\title{
ÜBER DEN
}

\section{INTERMITTIERENDEN EXOPHTHALMUS}

(EXOPHTHALMIE A VOLONTE; ENOPHTHALIIE E' EXOPHTHALMIE ALTERNANTES).

$\operatorname{VON}$

A. VOSSIUS IN GIESSEN.

Mit 2 Abbildungen auf Tafel XIX. 
Der in der Tiefe der Orbitalpyramide im Canalis opticus durch Vermittelung des Sehnerven befestigte Augapfel wird im vorderen Abschnitt der Augenhöhle durch die geraden und schiefen Augenmuskeln, speziell aber durch einen besonderen bindegewebigen Apparat in der Schwebe erhalten. Merkel hat uns von dem Fascienapparat der Orbita und von seiner Bedeutung für die Stellung und Bewegung des Auges in seinem Handbuch der topographischen Anatomie eine eingehende Schilderung und in Fig. 159 ein sehr anschauliches Schema dieses Bandapparates geliefert; er hat gezeigt, dass derselbe die Augenmuskeln umbüllt und sie einerseits mit dem Bulbus, andererseits mit der Orbitalwand verbindet. Die Verbindung mit der Augenhöhlenwand stellen die Fascienzipfel der Augenmuskeln her, welche sich an drei Stellen inserieren: am lateralen Umfang in der Gegend unter der Sutura zygomatico-frontalis, an der medialen Seite in der Gegend des Thränenbeines und oben innen an der Trochlea.

Am untern Umfang des Auges fehlt eine solche Verbindung des Fascienapparates mit der Orbita; hier besteht nur ein quer unter dem Bulbus verlaufender Fascienzug, welcher den am vordern Umfang des Auges von der Fascie gebildeten Bindegewebsring vervollständigt und verstärkt. Dieser Abschnitt des Fascienapparates ist besonders fest und so eingerichtet, dass der Bulbus auf ibm wie auf einer Schlinge ruht. Lockwood hatte 
diesen Teil deshalb auch Ligamentum suspensorium oculi benannt. Derselbe wird gebildet von den beiden sehr kräftigen Fascienzipfeln des Rect. inferior und erheblich verstärkt durch die rom Obl. inferior gelieferten Fascienteile, welche dem Ver. lauf des Obl. inferior entsprechend zu einem derben Bindegewebspolster verwachsen, welches gerade unter dem Bulbus liegt.

Dieser Fascienapparat stellt einerseits eine Hemmungsvorrichtung für die Wirksamkeit der Augenmuskeln auf die Bewegungen des Augapfels, andererseits eine wichtige Fixiervorrichtung des Bulbus selbst dar. Wenn man die Fascienzipfel durchschneidet, so kann das Auge in die Orbita zurücksinken.

Die Achse des Augapfels fällt nicht mit derjenigen der Augenhöhle zusammen, sondem sie schneidet dieselbe unter einem Winkel ron 30-45 Grad $^{1}$ ), je nach der Divergenz der Augenhöhlen. Sein Mittelpunkt liegt nach Merkel einige Millimeter lateralwärts von dem Mittelpunkt der Gesichtsöffnung der Orbita, so dass der Bulbus im ganzen mehr der äusseren als der inneren Wand der Augenhöhle genähert ist. Die Lage des Hornhautscheitels zu dem Augenhöhlenrand ist eine individuell sehr verschiedene. H. Cohn hat eingehende Messungen über die Prominenz der Augen mittelst eines besonderen Exophthalmometers bei 427 Individuen verschiedenen Alters, Geschlechts und Refraktionszustandes angestellt und als Ausgangspunkt derselben diejenige Stelle des oberen Augenhöhlenrandes gewählt, welche senkrecht über der Mitte der Pupille des in die Ferne blickenden Auges steht. Er fand, dass der Hornhautscheitel den genannten Punkt überragen (positive Prominenz), oder hinter ihm zurückstehen kann (negative Prominenz). Die Resultate schwankten zwischen $\mathrm{P}-10 \mathrm{~mm}$ und $\mathrm{P}+12 \mathrm{~mm}$, meist zwischen $-5 \mathrm{~mm}$

1) Diese Werte habe ich nach den Messungen von Weiss über den Winkel, welche beide Sehnerven mit einander bilden, angegeben. $D_{a}$ die Sehnerven nahezu in der Richtung der Augenhöhlenachse verlaufen, dürfte jene Angabe ungefähr zutreffen. 
und 0. Eine absolute Grenze zwischen physiologischer und pathologischer Prominenz kann hiernach nicht existieren; ein sicheres Urteil über eine pathologische Prominenz des Bulbus kann man durch diese Messungen nur gewinnen, wemn man die Lage beider Augen mit einander vergleicht und regelmässig zu verschiedenen Zeiten bestimmt. Aber auch der Vergleich der Stellung beider Augen giebt durchaus nicht immer einen zuver. lässigen Anhalt für die Annahme einer pathologischen Prominenz; denn unter 427 Personen gab es nur 82 mit gleich grosser $\mathrm{P}$ beider Augen. In 8 Fällen war $\mathrm{P}$ grösser als $\mathrm{O}$ und in $74 \operatorname{lag} \mathrm{P}$ gleich weit hinter dem zugehörigen Punkt des oberen Orbitalrandes. Bei allen übrigen Personen existierten zwischen rechtem und linkem Auge Differenzen von $1 / 2$ bis $8 \mathrm{~mm}$. Unterschiede von $1-3 \mathrm{~mm}$ sollen recht häufig sein und sich durch den Augenschein noch gar nicht bemerkbar machen, wemn $P$ negativ ist. Ist $\mathrm{P}$ positiv, so fallen grössere Differenzen beim ersten Anblick schon viel eher auf.

Alter und Geschlecht sind nach Co hns Untersuchungen ohne nennenswerten Einfluss auf die Prominenz der Augen, dagegen erwies sich dieselbe bis zu einem gewissen Grade abhängig von ihrem Brechzustand. Die Myopen lieferten die bedeutendste Prominenz; bei Hypermetropen war P meist negativ, indessen kam auch bei Myopie negative $P$. vor.

Abgesehen von dem Refraktionszustand ist die Lage des Auges zur Gesichtsöffnung der Augenhöhle aber noch abhängig von der Menge des Orbitalfettzellgewebes und gewissermassen auch von dem Füllungszustande der Blutgefässe, speziell der Venen. Bei fettreichen Personen stehen die Augen mehr vor als bei fettarmen. Bekannt ist das Einsinken der Augen (Enophthalmus) beim Hungerzustand, bei Kachexie nach oder während schwerer Krankheiten. Himly schreibt: „Bei Menschen mit kurzem Hals, apoplektischem Habitus, bei Säufern, während eines apoplektischen Anfalles, bei Erstickungszufällen, nach heftigen 
Körperanstrengungen (bei Neugeborenen, die schwer geboren wurden (Demours), treten die Augen leicht ein wenig hervor, aber auch leicht wieder zurück ....." Ferner beim Enophthalmus. „Der geringste Grad rührt von Verminderung des Fettpolsters der Orbita her, und folgt auf lange Trauer, Hunger, Auszehrung, bedeutenden Säfteverlust; totenäbnlich ist er bei der Cholera." A rlt äussert sich folgendermassen: „Von Natur aus bald tiefer bald flacher liegend, tritt er momentan bei erhöhtem Gefässturgor stärker hervor; bei reichlichem Säfteverluste (Hämorrhagie, Diarrhöe u. dergl.) merklich zurück, wogegen mechanische Hyperämie in dem retrobulbären Fettpolster mehr eine habituelle stärkere Vorlagerung (Glotzauge), starke Abmagerung aber ein mehr weniger auffallendes Zurücksinken in die Orbita (Hohlauge) bewirkt." Das Auge kann unter diesen Umständen wegen seiner Fascienverbindungen nur bis zu einem gewissen Grade in die Orbita zurücksinken; bei zunehmender Abmagerung vertieft sich die Tarsoorbitalfalte des Lides durch den Einfluss des äusseren Luftdruckes und täuscht hierdurch ein stärkeres Einfallen der Augen vor. Enophthalmus kommt, abgesehen von Sympathikus. lähmung, noch vor nach Verletzungen durch Fettschwund infolge Kompression des Orbitalzellgewebes bei Kontusionen (Nieden) oder durch Narbenzug.

Über den Einfluss der Blutgefässfüllung auf die Prominenz der Augen unter physiologischen Verhältnissen hat Donders sehr eingehende Untersuchungen angestellt. Er fand eine Ausdehnung der extra- und retrobulbären Gefässe (Venen) bei forciertem kräftigen Exspirationsdruck und sah, wenn der Exspirationsdruck eine Zeit lang anhielt, den Augapfel deutlich nach vorn treten, während das Gesicht rot und angeschwollen wurde. Bei jungen Menschen schien diese Veränderung geringer zu sein als bei älteren. Als Maximum beobachtete er bei einem 42 jährigen ein Vortreten um $1,45 \mathrm{~mm}$, im Mittel von 5 Fällen um 1,1 mm. Die Augenlider sind nach Donders im stande 
dieser respiratorischen Ausdehnung der Orbitalvenen entgegenzuarbeiten. Erweiterung der Lidspalte und fortgesetzter Exspirationsdruck zusammen bewirken ein stärkeres Vordringen der Augen. Bei jedem Nicken tritt der Bulbus in die Orbita zurück; sein Zurückweichen kann ungefähr $1 / 2 \mathrm{~mm}$ betragen. Dasselbe findet statt bei langsamer, willkürlicher Verengerung der Lidspalte; wenn man dann aber die Lidspalte wieder öffnet, während der Blick unverrückt auf denselben Punkt gerichtet bleibt, so tritt der Augapfel wieder gleichmässig vor. In jenem Fall fand Donders ein Zurückweichen um 0,41 bis $0,66 \mathrm{~mm}$, in diesem Fall ein Hervortreten um 0,37 bis $0,86 \mathrm{~mm}$. Diese Beobachtungen sind von hohem Wert für das Verständnis des Zustandekommens und des klinischen Krankenbildes des intermittierenden Exophthalmus. Jene Erscheinungen des Vortretens des Auges können durch nichts Anderes als durch eine Ausdehnung der Venen in der Augenhöhle beziehungsweise durch eine Gegenwirkung von seiten der Augenlider erklärt werden. Bei dem Mangel der gewöhnlichen Stützung des Bulbus durch die Augenlider kann die Ausdehnung der retrobulbären Venen allmählich einen hohen Grad erreichen; es können Varicen die Veranlassung für einen Exophthalmus abgeben, sagt Donders. So kann bei Keuchhusten ebenfalls Exophthalmus entstehen, wie Gunning auf einem Ophthalmologen-Kongress in Heidelberg berichtet hat, und zwar nach eigenen Beobachtungen durch retrobulbären Bluterguss.

Der Schluss und Druck der Augenlider bei erhöhtem Exspirationsdruck ist eine associierte Wirkung. Von einem Reflex auf eịn Schmerzgefühl, der sich an den Augen offenbaren soll, kann nach Donders anfänglich keine Rede sein. Erst später, bei anhaltendem Husten z. B., kann sich derselbe geltend machen. Aber das Gesamte der Bewegung, sowohl die des Exspirationsdruckes als die der Augenlider, kann ebenso gut durch Reflex (Husten, Niesen) als in Verbindung mit dem Willen 
(Blasen, Pressen, Schreien) oder automatisch mit dem Gemütsleben (Lachen, Schreien etc.) hervorgebracht werden.

Durch eine abnorme Füllung der Orbitalvenen ist ebenfalls nur die Protrusion $\%$ erklären, welche $H$. Cohn bei einem 17 jährigen, blühenden Mädchen während der Menstruation beobachtete; der Exophthalmus trat regelmässig mit einem Dickerwerden des Halses gleichzeitig auf und betrug $1^{1 / 2}$ bis $4 \mathrm{~mm}$. Eine von Sattler und Richter citierte, angeblich im Surgical dictionnary in dem Artikel "Exophthalmie" von Cooper mitgeteilte ähnliche Beobachtung habe ich an der bezeichneten Stelle nicht auffinden können. Durch Anschwellung der Orbitalvenen muss auch meines Erachtens der Exophthalmus in einem Fall von Louis, den Stellwag erwähnt hat, seine Erklärung finden. - Bei einem 3 jährigen Mädchen entstand mit der Auftreibung des Bauches Exophthalmus und verschwand mit dem Zurückgehen des Unterleibes auf sein normales Volumen.

Der in physiologischen Grenzen schwankenden Protrusion der Augen gegenüber steht der Exophthalmus, welchen wir als Zeichen verschiedener Erkrankungen der Augenhöhle und ihres Inhalts bei Panophthalmitis, Phlegmone des Orbitalzellgewebes, Thrombophlebitis der Orbitalvenen, bei Sinusthrombose, bei pulsierendem Exophthalmus, schliesslich bei Neubildungen in der Augenhöhle z. B. dem Cavernom auftreten sehen.

Einen vom pulsierenden Exophthalmus ganz abweichenden Symptomkomplex haben ,jene Fälle, welche, obwohl Sektionsbefunde noch fehlen, auf eine einfache variküse Erweiterung der Orbitalvenen zurückzuführen sein dürften". Sattler hat über die einschlägigen Beobachtungen in dem Handbuch von Gräfe-Sämisch, Bd. VI, pag. 879 kurz zusammenfassend referiert und die Veranlassung in einer individuellen Disposition, einer gewissen Schlaffheit und Dehnbarkeit der Venenwandungen als Ausdruck einer lokalen Erkrankung derselben gesucht. Von dem pulsierenden Exophthalmus unterscheidet sich das Krank- 
heitsbild dieser Fälle wesentlich durch das einseitige Auftreten der Protrusion, durch das Fehlen der Pulsation und Geräusche, sowie aller Stauungserscheinungen in der Orbita und am Bulbus. Das charakteristischste Symptom besteht in einem Hervortreten des Augapfels aus der Augenhöhle bei gewissen Körperbewegungen z. B. beim Bücken des Kopfes, während in aufrechter Haltung oder Rückenlage diese Vortreibung nicht besteht. Man hat deshalb das Krankheitsbild intermittierenden Exophthalmus oder, da eine gewisse Abhängigkeit von dem Willen der Patienten nicht zu verkennen ist, Exophthalmie à volonté genannt.

In der älteren Litteratur finden wir vereinzelte und nur sehr unvollkommen beschriebene, meistens als Kuriositäten bezeichnete Fälle. Auf dieselben ist zuerst von Himly in seinen Krankheiten und Missbildungen des menschlichen Auges hingewiesen. In den neueren Lehrbüchern der Augenheilkunde werden sie noch kaum erwähnt - ein Zeichen, dass sie selten und noch nicht von den einzelnen Autoren beobachtet sind. Nur Panas widmet unserem Krankheitsbilde eine ausführliche Besprechung. Vielfach ist das Hervortreten des Auges gleichzeitig mit einer sichtbaren und fühlbaren Geschwulst in der Umgebung des Bulbus oder hinter demselben beschrieben; bisweilen wurde auch nur eine Geschwulst neben dem Auge bei gebückter Kopfhaltung ohne Lageveränderung des Auges gesehen. Sattler hat beide Eventualitäten in seiner 1880 erschienenen Monographie von einem gemeinschaftlichen Gesichtspunkt aus betrachtet. Die älteren Beschreibungen seien hier kurz angeschlossen. So beobachtete Andreae ein Mädchen, bei welchem, sobald dasselbe einige Minuten in gebückter Stellung blieb, zwischen dem Augapfel und dem oberen Orbital. rande äusserlich eine längliche, weiche, elastische Geschwulst, grösser als eine halbe Haselnuss sich erhob, welche, wenn die Person eine Zeit lang den Kopf wieder aufrecht gehalten hatte, grossenteils verschwand. Die Farbe der Lidhaut war unver- 
ändert, das Übel schmerzlos, das Sehvermögen aber getrübt, besonders bei gefüllter Geschwulst. Durch eine Operation wurde der Varix beseitigt.

J. A. Schmidt wurde zu einem 16 Tage alten Kind zugezogen, dessen linkes Auge mit einer eigentümlichen Affektion behaftet war. Wenn man dem Kinde während des Schlafes die Augenlider öffnete, bemerkte man im äusseren Winkel einen kleinen venösen Tumor, welcher im Moment des Erwachens hervortrat, bei der Inspiration sich ein wenig zurückzog, beim Schreien aber den Augapfel vordrängte. Dabei drängten sich die Lider vor und färbten sich blaurot. Öffnete man jetzt die Augenlider, so sah man den Bulbus protrudiert und zwischen ihm und dem untern Augenlid einen grossen Varix von halber Daumenlänge. Derselbe bestand aus mehreren Varicen von verschiedenem Umfang, welche sich in die Augenhöhle unterhalb der äussern Kommissur hineinerstreckten. Wenn sich das Kind eine halbe Viertelstunde ruhig verhielt, verschwand der Varix allmählich und mit ihm der Exophthalmus, so dass beide Augen schliesslich in demselben Niveau sich befanden. Man fühlte dann in dem äusseren Winkel eine Höhle, welche sich bøim Schreien füllte; es handelte sich offenbar um eine variköse Erweiterung der Orbitalvenen, speziell der Vena ophthalmica cerebralis, die durch Schreien und bei allen forcierten Respirationen anschwollen.

Verduc erwähnte einen jungen Maler, welcher sich eines Tages einer Gesellschaft ron Ärzten vorstellte, um wegen eines ungewöhnlichen Vorfalls seines Auges Rat einzuholen; denn in weniger als einer Stunde trat sein Auge mehr als sechsmal aus der Augenhöhle bis auf die Mitte der Backe hervor, um sich dam wieder in die Orbita zurückzuziehen.

Velpeau berichtete, dass ein 16jähriges. Mädchen zwischen Auge und oberer Augenhöhlenwand einen venösen Tumor ge- 
habt habe, welcher regelmässig beim Bücken des Kopfes hervorgetreten sei.

Mackenzie behandelte einen Mann an rheumatischkatarrhalischer Augenentzündung, welchem während des Tragens einer schweren Last auf dem Rücken das rechte Auge fünf Jahre zuvor vorgefallen war. Seit dieser Zeit soll das Auge regelmässig beim Bücken vorgetreten und wieder in die Augenhöhle zurückgetreten sein, sobald er den Kopf hob oder das Auge rieb. Im Augenblick des Vorfalls fühlte er, dass etwas die Augenhöhle erfülle und das Auge vordränge; ausserdem empfand er einen Schmerz in der Orbita, welcher sich nach merkuriellen Abführmitteln verringerte. Die Bewegungen des Auges waren ungehindert, das Sehvermögen beim Vortreten des Auges etwas gestört.

Parrish ) soll nach Sattler und Rich te reinen 16jährigen Ladenjungen gesehen haben, welcher beim Tauchen einen Stoss gegen die Augengegend erhalten hatte und bei aufrechter Körperhaltung einen varikösen Venentumor zeigte, der beim Bücken und durch Kompression der Vena jugularis beträchtlich anschwoll.

Auf die ausführlichen und sehr viel genaueren Beobachtungen einschlägiger Fälle von intermittierendem Exophthalmus aus der neueren Litteratur werde ich am Schluss meiner Arbeit in einer Tabelle näher eingehen; an dieser Stelle will ich nur noch kurz einige von $\mathrm{Himly}^{2}$ ) berührte ältere Beobachtungen von bemerkenswertem Auftreten eines Exophthalmus erwähnen. So hat Schenk erzählt, dass die Augen einer Frau bei einer schweren Geburt aus den Höhlen heraus getreten und nach vier Tagen erst wieder zurückgewichen seien; ferner hat Sch e $\mathrm{n}$ k von

1) Den Fall habe ich an der von Sattler und Richter angegebenen Stelle nicht auffinden können.

2) Himly, Bd. I, pag. 394 und 395 . 
einem 60 jährigen Manne berichtet, welchem ungefähr alle halbe Jahre die Augen auf die Wangen vorgefallen seien.

Etwas eingehender als $\mathrm{Himly}$ und bereits mit einem Hinweis auf die Orbitalvenen als Ursache der Lageveränderung des Auges in derartigen Fällen behandelte Stell wag die vorliegende Frage. Er schreibt: „Varicen werden nur selten in ansehnlicher Entwickelung beobachtet. Sind sie oberflächlich gelegen, so lassen sie sich schon durch ihr Aussehen leicht erkemnen. Wenn sie aber tiefer gelegene Venenstämme betreffen, so ist ihre Diagnose nicht immer so leicht. Sie bedingen dann in der Regel eine Dislokation des Bulbus, welche offenbar unter dem Einfluss der Cirkulation steht, indem alle jene Umstände, welche eine vermehrte Blutzufuhr zu dem Kopf und in specie zum Auge bedingen, oder den Blutrückfluss behindern, die Vortreibung und etwaige seitliche Ablenkung des Augapfels steigern, während unter den entgegengesetzten Verhältnissen die Dislokation sich vermindert und durch allmählichen Jruck von aussen her wohl auch aufgehoben werden kann. Der völlige Mangel der Pulsation unterscheidet den Varix von dem Aneurysma."

Die erste gründlichere Bearbeitung der Fälle von intermittierendem Auftreten und Verschwinden eines Exophthalmus bei verschiedener Kopf- und Körperhaltung verdanken wir Dupont in seiner im Jahre 1865 erschienenen These „Des tumeurs de l'orbite formées par du sang en communication avec la circulation veineuse intracranienne." Dieser Autor berichtet zunächst über vier Fälle (Mackenzie, Verduc, J.A. Schmidt, Mazel et A. Boniface d'Anduze), ferner über eine Beobachtung von Foucher, dessen Patient von Nelaton auf operativem Wege dauerud geheilt wurde. Er entwirft eine genaue Symptomatologie, schildert den Verlauf, die Diagnose und Ätiologie dieser Fälle und erwähnt eine von Velpeau citierte Beobachtung Salmuths von dem Hervortreten des Auges bei einem Epi- 
leptiker während des Anfalls. Zur Erklärung des Exophthalmus greift er auf eine Anomalie der Orbitalvenen zurück; er führt bereits ein Anschwellen des Exophthalmus resp. der Tumoren bei Kompression der Jugularvenen, sowie bei tiefer Exspiration an - eine Erscheinung, welche ihre Erklärung in den Eingangs dieser Arbeit geschilderten physiologischen Beobachtungen von Donders findet - er bespricht genau den Einfluss des Bückens auf das sichtbare Hervortreten und die Grösse der Tumoren, sowie auf die Stärke des Exophthalmus und teilt die Fälle in zwei Gruppen ein, in solche, bei welchen der Exophthalmus ohne nachweisbaren Tumor eintritt, und in solche, bei welchen ein Tumor ohne Exophthalmus beobachtet werden kann.

Nach dem von Sattler gelieferten kurzen zusammenfassenden Referat über alle bis zum .Jahr 1880 beschriebeneu Beobachtungen dieser Art von intermittierendem Exophthalmus infolge variköser Erweiterung der Orbitalvenen hat dann Y vert in einer im Jahre 1881 im Recueil d'Ophthalmologie veröffentlichten Abhandlung ,Tumeurs veineuses de l'orbite en communication directe avec la circulation veineuse intracranienne" sich ausführlicher mit dieser Frage beschäftigt. Abgesehen von den vier Fällen Duponts bespricht er zwei neuere und eine eigene Beobachtung. Er macht dieselbe Einteilung wie Dupont: 1. in äusserlich sichthare Tumoren, welche sich bei Kopfneigung und Anstrengungen vergrössern, bei Rückenlage verschwinden oder durch geringen äussern Druck sich ganz resp. teilweise reponieren lassen - hierher rechnet er z. B. Schmidts Fall; 2. in intermittierenden Exophthalmus ohne äusserlich sichtbaren Tumor wie in den Fällen von Mackenzie, Grüning, Vieusse.

Dieser Gruppierung von Dupont und Yvert gegenüber steht eine Einteilung aller Fälle in drei Gruppen durch Sergent, auf dessen Abhandlung aus dem Jahr 1893 ich nummehr etwas eingehender zurückkommen muss, da sie abgesehen von einer neuen Beobachtung eine sehr ausführliche und bis heute 
völlig massgebende Übersicht über die Symptomatologie, Ätiologie, Genese und Differentialdiagnose unseres eigenartigen Krankheitsbildes bringt. Sergen $t$ unterscheidet folgende drei Gruppen:

1. Äusserlich sichtbare Tumoren ohne Exoph. thalmie - hierher zählt er die Fälle von Foucher, Mazel et Boniface, Yvert, Le Moine, Chisolm ${ }^{1}$ ).

2. Äusserlich sichtbare variköse Tumoren mit intermittierendem Exophthalmus; hierher gehören die Fälle von Schmidt und Magnus.

3. Intermittierender Exophthalmus ohne nachweisbare Tumoren in der Orbita; diese Gruppe umfasst die Fälle von Mackenzie, Grüning, Vieusse und von Sergent selbst.

Diese Einteilung entspricht vollständig den auch später noch festgestellten klinischen Thatsachen und dürfte, wenngleich uns auch noch die Bestätigung durch Sektionen fehlt, immerhin in Zukunft massgebend bleiben. Was allein bemängelt werden muss, ist die unvollständige Litteraturangabe in Sergents sonst so verdienstvoller Arbeit. Diese Lücke auszufüllen, soll der Zweck meiner Publikation sein, welche zunächst noch zwei eigene Beobachtungen und im Anschluss daran eine tabellarische Zusammenstellung aller bisher publizierten einschlägigen Fälle bringen wird. In der Tabelle habe ich einzelne kasuistische Mitteilungen nicht verwertet, von welchen es mir fraglich schien, ob sie in diese Kategorie von intermittierendem Exophthalmus

1) Der von Le Moine beschriebene Fall gehört gar nicht in diese Rubrik; denn es handelt sich um ein kavernöses Angiom der Haut und Bindehaut des oberen Lides, welches durch mehrfache Ignipunkturen mit Ferrum candens zerstört wurde. Der Fall von $\mathrm{Ch}$ isolm bietet aber insofern eine Analogie zu unserem Krankheitsbilde, als in dem unteren Lide eine seit 18 Jahren langsam entstandene Geschwulst erst bei gebückter Kopf- und Körperhaltung sehr deutlich hervortrat, in Rückenlage und bei aufrechter Kopfhaltung verschwand. 
hineingehören. So hat von Santen einen Fall von hochgradigem Exophthalmus durch spontane Hämorrhagie in die Orbita mit absoluter Amaurose beschrieben. Der ophthalmoskopische Befund war anfänglich normal, später bestand Optikusatrophie. Der Exophthalmus ging rasch zurück, wiederholte sich aber im nächsten Jahre ohne gleichzeitige Sugillation der Umgebung des Auges wie das erste Mal. Durch Bücken des Kopfes konnte ein Hervortreten des Auges und Anschwellung der Gegend der Fascia tarso-orbitalis hervorgerufen werden, die nachher immer wieder rasch verschwand. Leber führt diesen Fall bei den Erkrankungen des Sehnerven im Handbuch von Gräfe-Sä misch Bd. V, pag. 805 an und nimmt einen kavernösen Tumor der Orbita als Ursache des Leidens an. Ich will dieser Erklärung nicht entgegentreten; auffallend und im Widerspruch mit den anderen Beobachtungen von kavernösen Orbitalgeschwülsten ist aber der Unstand, dass der anfangs eingetretene Exophthalmus sich wieder ganz zurückbildete und später nur intermittierend beim Bücken des Kopfes eintrat. Von den Fällen von intermittierendem Exophthalmus durch variköse Erweiterung der Orbitalvenen unterscheidet er sich durch die absolute Amaurose infolge Sehnervenatrophie. In dem Fall von L. Mayer bildete sich das Leiden an einern durch Optikusatrophie erblindeten Auge aus.

Ganz dunkel hinsichtlich ihrer Ätiologie ist eine Beobachtung von Gruss, bei welcher der Autor ein akutes Ödem des retrobulbären Gewebes annahm. Mir selbst scheint in diesem Fall als Ursache des Leidens eine Blutstauung im Gebiet der Vena ophthalmica superior sehr wahrscheinlich zu sein. In der Nacht entstand bei einem kräftigen, in der Mitte der Dreissiger stehenden Manne ein sehr starker rechtsseitiger Exophthalmus begleitet von einem beträchtlichen Ödem des Lides. Durch die Zerrung der Trigeminusfasern bestand eine heftige Neuralgie. Bald gesellten sich dazu heftiges Erbrechen, das den Charakter 
des cerebralen Erbrechens hatte, Schwindelgefühl, Pulsverlangsamung bis auf 40 in der Minute. Die Beweglichkeit des Bulbus der erkrankten Seite war nur in so weit gehemmt, als dies die Protrusion desselben bedingen musste. Die Pupillen reagierten gegen Licht vollkommen prompt. Des Morgens liess das Erbrechen nach, die Pulsfrequenz hob sich, die Neuralgie besserte sich, der Exophthalmus fing an zurückzugehen. Am Abend waren die Erscheinungen subjektiv und objektiv geschwunden. Nach ca. 14 Tagen trat der Anfall infolge einer Aufregung wieder auf. Hofrat v. Bamberger stellte die Diagnose auf ein akutes retrobulbäres Ödem und empfahl mit Rücksicht auf die Adipositas des Patienten eine Marienbader Kur mit Diät, welche gewissenhaft durchgeführt wurde. Seither stellten sich noch Anfälle geringeren Grades ein, bei denen die cerebralen Erscheinungen sehr gering waren und das Erbrechen ganz ausblieb; sie kamen einmal infolge von psychischer Autregung, zweimal infolge von physischer Überanstrengung zu stande. Seit dem letzten Anfall waren zwei Jahre vergangen, als der Autor darüber berichtete. Liegt es hier nicht nahe wegen der vollständigen schnellen Ausgleichung des recidivierenden Exophthalmus bei Begirın des Leidens mit cerebralen Symptomen an eine Affektion des Sinus cavernosus mit vorübergehender Stauung des Blutes im Bereich der Vena ophthalmica superior zu denken?

Was das klinische Krankheitsbild des intermittierenden Exophthalmus ohne nachweisbaren Tumor hinter dem Bulbus anlangt, so kann ich darüber in Übereinstimmung mit Sergent nach den vorliegenden Beobachtungen folgendes berichten.

In einer Reihe von Fällen tritt das bei aufrechter Kopfhaltung oder bei Rückenlage normal stehende Auge auf einer Seite ohne nachweisbare Veranlassung beim Bücken, bei Körperanstrengungen, bei angehaltener tiefer Exspiration oder bei Kompression der Jugularvenen um mehrere Millimeter aus der Orbita 
hervor, ohne dass die Patienten dabei immer nennenswerte Schmerzempfindungen haben, abgesehen von dem Gefühl des Vorfalls, der Schwellung des Augapfels. Die Protrusion erfolgt oft nicht in gerader Richtung nach vorn, sondern der Bulbus ist dabei meist nach aussen und unten abgelenkt, ohne dass eine Muskellähmung oder eine stärkere Bewegungsbehinderung besteht. Diese Vortreibung wird nur ermöglicht durch die S förmige Krümmung des Sehnerven; sehen wir doch bisweilen bei Raufereien, worauf schon v. Arlt hingewiesen hat, eine Luxation des Auges zwischen die Lider eintreten, ohne dass dadurch das Sehvermögen geschädigt wird. Beim Aufrichten des Kopfes und in Rückenlage, beziehungsweise durch gelinden Druck, selbst durch einen kräftigen Lidschluss gleitet der Augapfel sofort wieder in seine frühere Lage zurück. An dem vorgefallenen Auge fühlt man weder eine Pulsation noch hört man jenes eigentümliche Geräusch über dem Bulbus und in dessen Umgebung wie beim pulsierenden Exophthalmus. Der intermittierende Exophthalmus ist und bleibt ferner immer nur einseitig; Sehstörungen treten dabei kaum je auf, ebensowenig lassen sich mit dem Augenspiegel während der Exophthalmie irgend welche Veränderungen der Gefässe im Augenhintergrund wahrnehmen. Hinter oder neben dem Bulbus kann unter Um. ständen ein venöser Tumor sicht- und fühlbar sein oder erst bei gebückter Kopthaltung hervortreten; in einer grossen Reihe von Fällen vermisst man aber auch solch eine Geschwulst.

Mehrfach, und ganz speziell bei den Fällen ohne nachweisbaren Tumor in der Orbita, ist berichtet, dass das vorfallende Auge für gewöhnlich eigentümlich in die Augenhöhle eingesunken erschien, dass also ein gewisser Grad von Enophthalmus bestand, welcher eine gewisse Ähnlichkeit mit einem künstlichen Auge bedingt insofern, als auch die physiologische TarsoOrbitalfalte des obern Lides erheblich vertieft ist und zuweilen Ptosis besteht. Die Ptosis kommt häufig auch erst beim Bücken 
des Kopfes mit dem Exophthalmus gleichzeitig zur Entwickelung und schwindet beim Aufrichten des Kopfes. In einzelnen Fällen sind Bewegungsstörungen an dem befallenen Auge beobachtet. Gelegentlich war die Pupille desselben etwas erweitert.

Der Exophthalmus tritt meist ganz zufällig und plötzlich bei jugendlichen oder erwachsenen Personen während der Arbeit bei gebückter Kopf- oder Körperhaltung auf und wird dann regelmässig bei Einnahme dieser Position wieder beobachtet. Zuweilen treten Blutungen in die Lider oder Orbita durch Bersten eines Blutgefässes ein, welche den Exophthalmus zunächst vorübergehend erzeugen, später aber intermittierend sein lassen. Es handelt sich fast immer um früher gesunde Individuen, sowohl männlichen als auch weiblichen Geschlechts. In einem Fall von Gessner entwickelte sich der intermittierende Exophthalmus direkt im Anschluss an den Eintritt der Menses, bei dem ja wie früher angegeben, nach den Beobachtungen von Cohn ein Exophthalmus nichts Ungewöhnliches und die Folge von stärkerer Füllung der Orbitalvenen sein soll. Bei einzelnen Patienten soll dem Auftreten der intermittierenden Exophthalmie eine Verletzung verschieden lange Zeit voraufgegangen sein. --Männliche Personen sind etwas häufiger befallen gewesen als weibliche; rechtes und linkes Auge haben keinen Unterschied in der Häufigkeit der Anomalie erkennen lassen.

Die Prognose ist eine gute; denn selbst bei mehrere Jahrzehnte langem Bestehen des Leidens hat sich weder ein nennenswerter kosmetischer Nachteil, noch eine Störung der Beweglichkeit oder des Sehvermögens gezeigt. Einer Rückbildung ist das Leiden aber nicht fähig.

In Übereinstimmung mit Dupont, Sattler und Yvert führt Sergent den intermittierenden Exophthalmus auf eine variköse Affektion der Orbitalvenen, speziell der V. ophthalmica superior zurück. Als prädisponierende Ursache sieht er eine kongenitale lokale Anlage der Venen, möglicherweise eine 
dünne und weniger widerstandsfähige Wandung derselben auf der betreffenden Seite an; dabei könnte eventuell auch eine Anomalie des Skeletts in der Fissura orbitalis superior beim Durchtritt der Vene die Veranlassung zur Behinderung der Blutcirkulation abgeben. Begünstigend auf das Zustandekommen der Venenerweiterung und der Lageveränderung des Auges könne ferner eine Abnahme des Orbitalfettzellgewebes und eine Auflockerung des Fascienapparates des Bulbus einwirken. Das Fehlen der Klappen in den Orbitalvenen erleichtere die Blutstauung.

Die Diagnose des intermittierenden Exophthalmus macht keine Schwierigkeiten, wenn man das Fehlen der Pulsation, der Geräusche und die Steigerung der Protrusion des Bulbus durch die Kompression der Jugularvenen berücksichtigt. Differentielldiagnostisch kämen vor allem der pulsierende Exophthalmus und das Angioma cavernosum der Orbita in Betracht. Auch bei dem pulsierenden Exophthalmus kann zuweilen bei gebückter Stellung des Oberkörpers, bei gewaltsamer Exspiration und durch Kompression der Jugularvenen ein stärkeres Hervortreten des Auges konstatiert werden, wie in einem Fall von de Vincentiis. Pulsation, hörbare Geräusche, die bei aufrechter Körperstellung und Rückenlage, sowie durch Druck nie völlig zu beseitigende Exophthalmie, die hochgradigen Stauungserscheinungen am Bulbus und in den Netzhautgefässen, sowie Sehstörungen werden immer wichtige diagnostische Hilfsmittel abgeben. In dem Fall von de Vincentiis lag eine angeborene Erweiterung der V. ophthalmica bei weiter Kommunikation derselben mit dem Sinus cavernosus dem Leiden zu Grunde.

Grössere Schwierigkeiten kann man hinsichtlich der Differentialdiagnose mit einem retrobulbären Cavernom haben. Hier wird besonders das konstante Bestehen eines gewissen Grades von Exophthalmus bei Rückenlage und bei aufrechter Stellung, das langsamere und unvollständige Zurücksinken des protrudierten Bulbus beim Übergang aus der gebückten in die auf- 
rechte oder Rückenlage und der Nachweis eines mehr minder kompressibeln Tumors hinter dem Auge von Ausschlag gebender Bedeutung sein. Schliesslich treten bei stärkerem Wachstum der Geschwulst durch Kompression des Sehnerven Sehstörungen ein, welche man bei der Exophthalmie à rolonté in der Regel vermisst. Zum Beweise für die grosse Ähnliclıkeit des klinischen Krankheitsbildes bei retrobulbärem Cavernom und intermittierendem Exophthalmus führt Sergent einen Fall von Dolgenkow aus dem Jahr 1886 an, bei dem 14 Jahre zuvor ein Trauma stattgefunden, vor 7 Jahren zum ersten Mal der Exophthalmus bemerkt, eine Luxation des Auges vor 3 Jahren während eines Hustenanfalls aufgetreten war und durch Bücken, Anhalten des Atems, sowie durch Kompression der V. jugularis interna eine Steigerung des Exophthalmus erzielt wurde, während in Rückenlage nur eine unbedeutende Abnahme der Protrusion des Auges beobachtet werden konnte.

Einer der letzten Autoren in der Litteratur über den intermittierenden Exophthalmus, P. V. Richter, stellt sich im Anschluss an einen Fall aus der Münchener Augenklinik ganz auf den Standpunkt Sergents hinsichtlich der Gruppierung und Genese der Exophthalmie à volonté; einer Beobachtung ron Gräfes und von Grüning, welch' letztere Sergent als der seinigen sehr ähnlich bezeichnet, weist er indessen entschieden mit Recht einen Platz an unter der Rubrik ,Exophthalmus intermittens mit gleichzeitigem Cavernoma orbitae". Bei dem Fall v. Gräfes stützt er sich auf des Autors eigene Angaben - scharf gegen gesundes Gewebe sich abgrenzende Geschwulst, Bezeichnung derselben als cavernöser Tumor und Sichtbarkeit einer Prominenz im äusseren Augenwinkel bei aufrechter Körperhaltung -; Richter nimmt dabei einen Sitz des Cavernoms ausserhalb seiner Prädilektionsstelle an und erklärt das Fehlen eines dauernd bestehenden Exophthalmus durch Schwund des Orbitalfettzellgewebes. In dem Fall Grünings spricht die 
Exophthalmie bei aufrechter Körperhaltung, das Vorhandensein von Kompressionserscheinungen an den Retinalgefässen und am Optikus, sowie die Unbeweglichkeit des protrudierten Auges nach der Ansicht Richters ebenfalls für die Annahme eines Cavernoms; eine normale Lage des Bulbus durch Druck desselben gegen das Orbitalpolster könne leicht vorgetäuscht worden sein.

Ob die Erklärung Richters zutrifft lässt sich natürlich nicht mit absoluter Sicherheit entscheiden; man könnte auch durch variköse Venenerweiterung um den Optikus, Gefässveränderungen in der Netzhaut und eine Alteration des Sehnerven selbst eintreten sehen. Zur Stütze dieser Annahme giebt es in der Litteratur anatomische Befunde. Ich verweise nur auf die Abbildungen von de Wecker, aus denen hervorgeht, wie hochgradig die Erweiterung der Orbitalvenen werden kann, und dass derartige Venen auch auf den Sehnerven einen Druck auszuüben im stande sind, lässt sich nicht von der Hand weisen. Welcher kolossalen Erweiterung die Venen bei pulsierendem Exophthalmus fähig sind, mögen folgende Zahlen beweisen. In einem Fall von Gendrin war die V. ophthalmica superior 9, die V. lacrymalis 6-7 mm dick; in einem Fall von Aubry war die V. ophthalmica superior kleinfingerdick und zeigte auf ihrem Wege durch die Orbita zahlreiche Schlängelungen. Aus der älteren Litteratur seien ebenfalls einzelne Mitteilungen über starke Varikositäten der Orbitalvenen angefügt. So findet sich in Siebolds Chiron, Bd. 3, pag. 375 ff., folgender Fall beschrieben. Bei einem 15 jährigen Knaben, welcher im 5. Lebensjahr nach Blattern eine Entzündung beider Augen gehabt und darnach Flecken auf dem linken Auge zurückbehalten, bald nachher Bauchwassersucht überstanden hatte, war das linke Auge ohne Schmerz und Sehstörungen hervorgetreten. Eine dem Auge in der Augenhöhle zunächst gelegene Blutader hatte eine so grosse Ausdehnung angenommen, dass dadurch der 
Bulbus hervorgetrieben wurde. Nach einer teils durchs Messer, teils durch Ätzmittel vorgenommenen Zerstörung des Varix trat der Augapfel wieder in seine Normallage zurück. Siebold war mit dem bei der Operation anwesenden Anatomen Dr. $\mathrm{H}$ esselbach dahin übereingekommen, dass diese variköse Aus. dehnung in der Hirnaugenvene (V. ophthalmica cerebralis) ihren Sitz hatte; denn die fluktuierende, venöses Blut enthaltende, einem Bruchsack ähnliche Geschwulst nahm wie diese Vene am innern Augenwinkel ihren Anfang und verbreiterte sich schräg über den Sehnerv nach aussen.

M. J. A. Schön erwähnt in seinem Handbuch der pathologischen Anatomie des Auges, pag. 159, ebenfalls die Blutaderknoten der Orbitalvenen und schreibt: „Man hat sie zuweilen in den Venen des Auges und meistens durch sie einen Exoph. thalmus entstehen gesehen. So fand Morgagni (l. c. epist. 13 N. 6) Varikositäten in den Gefässen, die den Sehnerven umgaben und dadurch Amaurose herbeigeführt haben." Von einem Fall von wahrem Aneurysma beider Art. ophthalmicae mit pulsierendem Exophthalmus Guthries heisst es, dass die V. ophthalmica cerebralis sehr erweitert und da, wo sie durch die Fissura supraorbitalis durchtritt, durch eine bedeutende Anschwellung der vier geraden Augenmuskeln verschlossen war.

Schliesslich sei noch daran erinnert, dass v. Gräfe bei einem Fall von angeborenem Angiom der Orbita in einer Phlebektasie einen erbsengrossen Phlebolith nachgewiesen hat, und dass dieser Fall eine Analogie zu den Konkrementbildungen in varikösen Venen an anderen Körperstellen bietet.

Alle diese angeführten Thatsachen beweisen das Vorkommen variköser Venenerweiterungen an den Orbitalvenen und lassen die Möglichkeit zu, dass auch in dem Fall von Grüning der geringe Grad von Glotzauge am rechten Auge mit intermittierendem Exophthalmus durch hochgradige Venenektasieen veranlasst worden sein könnte. Mit Sicherheit lässt sich dies aber 
nicht behaupten; denn obwohl ein 'T'umor hinter dem Bulbus nicht nachweisbar war, ist das Vorhandensein eines solchen doch möglich. Der Fall ist zweifelhaft.

Zum Schluss meiner Litteraturübersicht muss ich noch auf eine wenn auch nur indirekt hierher gehörende Beobachtung eingehen, welche neuerdings Jocqs publiziert, auf "dilatation veineuse de l'orbite" zurückgeführt und auf eine Stufe mit einem von de Wecker früher veröffentlichten Fall gestellt hat. Die Analogie schien ihm um so näher zu liegen, da bei seinem Patienten gleichfalls ,par la compression extraorbitaire“, nämlich durch Druck auf die Gegend des inneren Augenwinkels sofort das Geräusch verschwand. Der Kranke selbst hatte diese Entdeckung gemacht; ja es genügte dazu sogar die Augenbraue zu reiben oder das Pince-nez etwas hoch in den Augenwinkel aufzusetzen. Was nun den Fall von Jocqs anlangt, so hatte der ó1jährige Patient keine Verletzung erlitten; er war ein Jahr zuvor eines Morgens mit einem eigentümlichen Geräusch im linken Ohr erwacht, welches ihn den ganzen Tag über, besonders aber Nachts sehr stark genierte. Zuerst konsultierte er einen Ohrenarzt, welcher nur geringe Rötung fand, darauf Jocqs, welcher eine geringe Protrusion des linken Auges, mehrere stark erweiterte und geschlängelte konjunktivale Venen, normale Augen- und Lidbewegungen konstatierte, weder Pulsation noch Geräusche mit der aufgelegten Hand wahrnehmen komnte. S war 1. Bei der Augenspiegeluntersuchung ergab sich eine mässige Erweiterung der V. centralis retinae. Die Venen der Lider und Stirn waren nicht erweitert. Kompression der Carotis war wegen grossen Fettreichtums am Halse schwer ausführbar, brachte aber das Geräusch zum Schwinden. Jocqs diagnostizierte ebenso wie Panas einen retrobulbären Gefässtumor. Im Verlauf der nächsten Zeit nahm der Exophthalmus noch etwas zu, blieb dann aber stationär, während das Geräusch sich verringerte, ja sogar für mehrere Tage verschwand, um dann wieder

Anatomische Hefte. 1, Abteilung. Xxvill bis xxx. Heft (9. Bd, Il. 1 bis 3). 15 
aufzutreten. Die Behandlung bestand in häufiger Kompression der Carotis. Am interessantesten und für die Diagnose der Art des fraglichen Tumors von Wichtigkeit war die Sistierung des Geräusches durch extraorbitären Druck. In de Weckers Fall geschah dies durch Druck auf die erweiterte V. supraorbitalis; bei dem Kranken von Jocqs war ein erweitertes venöses Gefäss nicht vorhanden, aber Druck auf die Gegend des inneren Augenwinkels, wo man die Wurzel der V. ophthalmica superior zu suchen hat, hatte denselben Erfolg d. h. die Unterdrückung des Geräusches. De Wecker nahm an, dass die Pulsation von der Arterie auf die erweiterte, ihr aufliegende Vene übertragen würde. Dieser Kompression der Arterie durch die erweiterte V. ophthalmica schreibt Jocqs die Entstehung jenes Geräusches zu. Komprimiere man nun die Wurzel der V. ophthalmica, behindere man die Blutzufuhr, so entleere sich die Vene; sie komprimiere die Arterie nicht weiter und das Geräusch höre auf. Die Entleerung der Vene sei jedoch nicht so hochgradig, dass der Exophthalmus beseitigt werde. Auf die Sistierung des Geräusches durch Kompression der Zuflüsse zur V. ophthalmica stützt Jocqs seine Diagnose der Dilatation der V. ophthalmica, die entweder durch eine Verletzung (Fraktur durch die Fissura supraorbitalis) oder durch eine Endophlebitis veranlasst werden könne.

Jocqs vergleicht diesen Fall ferner mit den Beobachtungen von intermittierendem Exophthalmus bei gebückter Kopfhaltung oder bei Kompression der Jugularvenen, obwohl dabei niemals ein Geräusch verzeichnet ist. Dass beim Kopfbücken das Blut sich in dem varikösen Hohlraum anhäufe, sei leicht zu begreifen. Bei Kompression der Jugularvene könne das Blut in den Hirnsinus oder in der V. facialis stauen, im letzteren Fall auch in der V. ophthalmica, mit der sie durch die V. angularis kommuniziere; auf diese Weise könne Exophthalmus entstehen. Ein Geräusch könne unter diesen Umständen zunächst fehlen 
und erst dann auftreten, wenn die Dilatation der V. ophthalmica so umfangreich geworden sei, dass die Art. ophthalmica komprimiert werde. Die Möglichkeit, dass die Erklärung von J ocqs zutrifft, kann ich nicht leugnen, indessen bleibt das Fehlen der Exophthalmie beziehungsweise das Vorhandensein von Enophthalmus bei intermittierendem Exophthalmus während aufrechter Kopf- und Körperhaltung unaufgeklärt, während in seinem Fall ein gewisser Grad von Protrusion des Auges bestand; man müsste denn einen erheblichen Fettschwund in der Orbita supponieren oder annehmen, dass die Dilatation der Venen bei dem intermittierenden Exophthalmus nur unbedeutend sei im Verhältnis zur Ausdehnung derselben bei seinem und de $W$ eckers Patienten. Wie sich in diesen beiden Fällen der Exophthalmus bei Kopfneigung und Kompression der Jugularvenen verhalten hat, ist aus der Publikation von Jocqs nicht ersichtlich. Mir scheint deshalb eine Konfundierung dieser Beobachtungen mit denen von intermittierendem Exophthalmus nicht angebracht zu sein; denn gerade der Vorfall eines in normaler Lage befindlichen oder sogar leicht eingesunkenen Auges bei gebückter Haltung des Kopfes und bei Kompression der Jugularvenen hat dem ganzen schon von Dupont und Sattler abgesonderten Krankheitsbilde ein ganz charakteristisches, differentiell.diagnostisch sehr wichtiges Gepräge verliehen. Übrigens hat bereits Sattler gegenüber dem Fall von de Wecker und dessen Erklärung des Zustandekommens der Pulsation sowie der Geräusche Stellung genommen in seiner Monographie über den pulsierenden Exoplthalmus und darin die Ansicht vertreten, dass die einfache Kompression der Arterie durch die erweiterte V. ophthalmica unmöglich dieses Krankheitsbild des pulsierenden Exophthalmus herrorrufen könne, dass vielmehr in dem Fall de Weckers voraussichtlich eine vielleicht nur unbedeutende und bei der Sektion nicht bemerkte Kommunikation zwischen der Carotis interna und dem Sinus caremosus bestanden labe. 
Bei dem Kranken von Jocqs kommt noch hinzu, dass die Kompression der Carotis das Geräusch zum Schwinden brachte und auch einen günstigen Einfluss auf das ganze Leiden gehabt haben soll, wie dies beim pulsierenden Exophthalmus ebenfalls beobachtet ist.

Was nunmehr meine beiden eigenen Beobachtungen anbelangt, so handelte es sich in dem ersten Fall, welcher am 2. September 1896 zur Klinik kam, um einen 39 jährigen Eisenbahnstationsassistenten. Derselbe hatte eine beiderseitige $\mathrm{H}_{y}$ permetropie r. $+1,0 \mathrm{D}, 1 .+1,25 \mathrm{D}$, normale Sehschärfe; und asthenopische Beschwerden, gegen welche ihm eine Brille verordnet wurde. Als ich im Fortgehen den Kranken sah, teilte mir mein Assistent Dr. Nieser mit, dass er bei dem Patienten eine Insuffizienz des R. superior von ca. $12^{\circ}$ gefunden und beim Bücken einen Exophthalmus beobachtet habe, welcher nach dem Aufrichten sehr schnell wieder schwand. Die rechte Gesichtshälfte sah dabei etwas gerötet und turgescent aus, wenn der Exophthalmus beim Bücken auftrat. Ich überzeugte mich von der Richtigkeit der Beobachtung, konnte den Fall indessen aus Mangel an Zeit nicht mehr genauer untersuchen. Herr II. versprach mir später noch einmal zu einer eingehenderen Besichtigung wiederzukommen, ist indessen nicht mehr erschienen. Auf eine schriftliche Anfrage hat er mir geantwortet, dass seine Augen früher immer von guter Beschaffenheit waren, dass er erst seit zwei Jahren ohne nachweisbare Veranlassung, möglichenfalls durch angestrengte Arbeit bei elektrischem Licht sein Augenleiden bekommen habe. Im Jahre 1886/87 habe er an Gelenkrheumatismus gelitten; zur Zeit sei er gesund bis auf einen starken Katarrh, der seit drei Wochen bestehe, ausserdem habe er, wie wohl alle seine Amtsgenossen, zeitweise mehr oder weniger an Hämorrhoiden zu leiden. Ein Tumor war in diesem Fall weder sicht- noch fühlbar, wenn der Patient stand oder sich 
bückte. Ich nahm damals eine variköse Erweiterung der Orbjtalvenen als Ursache an.

Ausführlicher und genauer ist meine Beobachtung und die Krankengeschichte von dem 2. Fall.

Der 24jährige Tapezier W. L. aus Wetzlar stellte sich mir am 30. November 1896 zum erstenmal in der Poliklinik vor wegen heftiger Schmerzen, welche ihn seit zwei Tagen in seinem rechten Auge befallen hatten und an einen eigentümlichen Anfall erinnerten, den er im Jahr 1891 in Marburg durchgemacht hatte. Nach seiner Aussage sei zu jener Zeit plötzlich nach einem heftigen Schmerzanfall das rechte Auge aus der Augenhöhle vorgetreten; dieser Vorfall sollte sich seit der Zeit regelmässig beim Bücken wiederholt haben.

Bei der Erhebung der genaueren anamnestischen Daten wusste er aus seiner Kindheit nur anzugeben, dass er einmal auf eine Flasche gefallen sei und von dieser Verletzung cine Narbe am Bauch unterhalb des Nabels zurückbehaiten habe. Sein Vater soll an Phthise gestorben sein. Im Jahr 1886 sei er selbst wegen Blutens aus Mund und Nase behandelt. Anfangs seien diese Blutungen sehr häufig, meist $10 \mathrm{mal}$ am Tage aufgetreten, später weniger olt, vor zwei Jahren etwa dreimal täglich und seit zwei Jahren hätten sich dieselben bis jetzt nicht mehr wiederholt. Im Jahr 1890 sei er zuerst wegen Lungenleidens in Marburg behandelt, u. a. auch mit Tuberkulineinspritzungen; während dieser Kur sei er im Februar 1891 in einer Nacht plötzlich unter Schmerzgefühl im rechten Auge erwacht und als er nach dem Auge fühlte, habe er ein Hervortreten desselben bemerkt. Am nächsten Tage sei wieder alles gut gewesen. Nach vier Tagen habe sich dieser Anfall wiederholt; er sei dabei schwindlig geworden, ausserdem solle das Auge blutunterlaufen gewesen sein. Infolge dieser Komplikation habe er sich in Narburg in der Augenklinik behandeln lassen. Ob er damals einen starken Husten gehabt habe, wusste er sich nicht zu ent- 
sinnen; aus Ermangelung einer anderen Ursache schrieb er den Tuberkulineinspritzungen die Hauptschuld an seinem Zustand zu. Wegen seines Auges soll er vom Militärdienst befreit worden sein. Frische ähnliche Anfälle sollen seit 1891 nicht vorgekommen sein, nur blieb ein Hervortreten des rechten Auges beim Bücken zurück, während bei aufrechter Haltung oder Rückenlage des Körpers die Stellung des Auges eine normale gewesen sei. Auch bei rechter Seitenlage, bei erschwertem Stuhlgang, während Obstipation und beim Coitus, sowie bei schweren körperlichen Anstrengungen trete das Auge regelmässig hervor.

Patient ist von mittlerer Grösse, schlank gebaut, hat einen schwachen Panniculus adiposus, frische Gesichtsfarbe, regelmässigen Puls. An seinem Herzen ergiebt die Untersuchung durchaus normale Verhältnisse; der Urin ist ebenfalls normal. Über der rechten Lungenspitze besteht noch eine geringe Dämpfung. Er hat in letzter Zeit weder Husten noch Hämoptoe gehabt. Keine Varicocele, keine sichtbaren Venenerweiterungen an Armen oder Beinen. Auf der Haut der Brust befindet sich über dem Processus xiphoideus ein gerstenkorngrosses, rundliches etwas prominentes Angiom; darüber sieht man in einigem Abstand noch zwei kleine stecknadelkopfgrosse, anscheinend in der Entwickelung begriffene, noch kaum prominente blutrot verfärbte Flecken, in der Entstehung begriffene Teleangiektasien. Die Haut der Wange ist leicht gerötet. In der Haut des rechten immeren Augenwinkels und unter dem rechten äusseren Augenwinkel sieht man eine etwas stärker ausgesprochene Vene. Bei gewöhnlicher aufrechter Stellung des Kopfes (s. Fig. 1) erscheint das rechte Auge etwas tiefer in der Augenhöhle liegend als das linke, und die Tarso-Orbitalfalte im oberen Augenlide etwas vertieft, so dass das Auge eine gewisse Ähnlichkeit mit einem Oculus arteficialis hat. Seine Beweglichkeit ist nach allen Richtungen normal, ebenso das Sehvermögen für Feme und Nähe und der Farbensinn. Bei der Augenspiegeluntersuchung be- 
merkt man am Rande der physiologischen Exkavation der im übrigen normaleu Papille einen deutlichen Venenpuls in physiologischer Breite. Die Venen erscheinen rechts vielleicht etwas stärker gefüllt als links, nicht geschlängelt, die Arterien von normalem Kaliber.

Für gewöhnlich erscheint der rechte Augapfel ebenso blass wie der linke. Senkt Patient den Kopf, noch mehr aber, wenn er den Rumpf in der Taille rechtwinklig nach vorn beugt, so bemerkt man bereits wach 7 bis 8 Sekunden eine eigentümliche Turgescenz und bläulichrote Verfärbung der rechten Gesichtshälfte (cfr. Fig. 2); das obere Augenlid schwillt an und senkt sich etwas, so dass die Lidspalte kleiner wird. Die vorher bereits sichtbaren, oben näher bezeichneten kleinen Hautvenen füllen sich wie ein paar Venen der Stirn sehr stark. Dabei tritt der Augapfel um fast $1 \mathrm{~cm}$ nach vorn vor (cfr. Fig. 2); er ist gleichzeitig etwas nach unten und aussen abgelenkt. Die Gefässe der Conj. bulbi sind ziemlich stark gefüllt. Nach 8-10 Sekunden beim Aufrichten lässt die Turgescen\% der Gesichtshaut und der Lider nach, der Augapfel blasst ab und es tritt wieder der geringe Enophthalmus ein. Beim Vortreten des Bulbus besteht weder Schmerz, noch eine Sehstörung, noch eine Behinderung der Beweglichkeit. Die Pupille bleibt normal weit, eine stärkere Füllung der Hintergrundsvenen oder lebhaftere Pulsation tritt nicht ein. Man fühlt weder noch sieht man während des Exophthalmus oder Enophthalmus hinter oder neben dem Bulbus einen 'Tumor oder Venenstränge. Ebenso wenig kann man ein Geräusch bei der Auskultation der Orbita und ihrer Umgebung hören.

Evident ist der Exophthalmus sowie die Turgescenz der Gesichtshaut und der Lider bei der rechten Seitenlage. In sehr geringem Grade ist dieses Symptomenbild durch Kompression einer Jugularvene zu erzeugen; bei Kompression beider Jugularvenen tritt der Symptomenkomplex viel deutlicher hervor, aber 
er erreicht nicht den hohen Grad wie beim Bücken des Oberkörpers. Übt man während des Bückens einen Druck auf das obere Lid in der Gegend der Trochlea aus, ohne den Bulbus zu berühren, so wird der Exophthalmus nicht so deutlich. Ist das Auge bei dem Bücken hervorgetreten und legt man dann wieder bei aufrechter Körperhaltung leise die Hand auf das rechte Auge, so fühlt man deutlich, wie dasselbe förmlich in die Augenhöhle zurückfällt. Eine einfache Orbikulariskontraktion genügt gleichfalls, um den Exophthalmus in den gewöhnlichen Enophthalmus umzuwandeln. Nach dem Aufrichten des Kopfes verschwindet übrigens die Divergenz des Auges zuerst.

Auf Grund dieses Befundes diagnostizierte ich einen intermittierenden Exophthalmus dexter durch variköse Erweiterung der Orbitalvenen bei gewöhnlich vorhandenem Enophthalmus infolge Abnahme des Orbitalfettes. Den Patienten demonstrierte ich in meiner klinischen Vorlesung und in der Sitzung der medizinischen Gesellschaft vom 8. Dezember 1896.

Nachträglich stellte ich noch bei Herm Geheimrat Mann. kopf und Herrn Kollegen Hess weitere Nachforschungen über den Befund bei dem Patienten während seiner Kur in den Marburger Kliniken an. Von Herrn Geh. Rat Mannkopf erhielt ich folgende Notizen aus dem Journale der Marburger Poliklinik, für die ich auch an dieser Stelle bestens danke.

20. X. 90. Vater an Phthise gestorben. Husten, Heiserkeit. R. Spitze leicht gedämpft, Atmungsgeräusch sakkadiert, verschärftes Exspirieren, leichtes Rasseln. Ord.: Kreosotpillen, Inhalationen.

18. XII. 1891. Pat. ist vom 27. XI. 90 bis Ende Januar 1891 mit Kochschen Injektionen behandelt worden; er hat stark darauf reagiert, ist auch während der Behandlung heiser geworden. In der Folgezeit hat sich Pat. sehr wohl gefühlt. Rechts hinten oben noch ganz leichte Abdämpfung, Atmungs. 
geräusch hier etwas abgeschwächt; links hinten oben etwas rauh, keine Rasselgeräusche.

29. I. 92. Das rechte Auge tritt vor beim Bücken. Besserung nach mehrfacher Faradisation des Auges; Anode auf das Lid gesetzt. Auf Wunsch: Kreosot, Leberthran.

26. II. Rechts hinten oben Atmungsgeräusch sehr abgeschwächt. Sonst nichts.

Ebenso bin ich Herrn Kollegen Hess zu grossem Dauk verpflichtet für die freundliche Überlassung der Krankengeschichte aus der Augenklinik, welche mir eine wichtige Aufklärung über die Entwickelung des Augenleidens brachte. - - Ich teile dieselbe mit gütiger Erlaubnis hier zum weiteren Verständnis für die Pathogenese des Zustandes mit. In Marburg war übrigens seiner Zeit die Diagnose auf periodischen Exophthalmus infolge von retrobulbärer Hämorrhagie (venöses Angiom der Orbita?) gestellt. Aus der dort erhobeneu Anamnese sei noch mitgeteilt, dass der Vater an Phthisis gestorben war, dass Patient selbst am 5. Februar 1891 in die Marburger Augenklinik recipiert wurde. Er hatte damals angegeben, dass er im Jahre 1886 wegen häufigen Blutens aus Mund und Nase behandelt, seit zwei Jahren lungenleidend gewesen und seit dem 17. XII. 90 deshalb mit Koch'schen 'Tuberkulininjektionen behandelt worden sei. Seit einem Jahr habe er öfters an diffusen Schmerzen im rechten Auge zu leiden gehabt; es habe ihm ferner den Eindruck gemacht, dass der Augapfel jedesmal, wenn er sich bücke, etwas hervortrete, ebenso bei starken Anstrengungen. Die Schmerzen seieu an Häufigkeit und Stärke sehr wechselnd gewesen, das Sehvermögen solle nicht gelitten haben. Seit etwa drei Wochen sollen die Schmerzen häufiger und stärker geworden sein, auch meinte er, dass seit der Zeit das rechte Auge dauernd etwas dicker sei, dass ferner das obere Lid etwas herabhing. Vor ca. acht Wochen nun stellten sich plötzlich ohne nachweisbare Ursache ungewöhnlich heftige Schmerzen ein, das Auge trat erheblich stärker vor, das obere 
Lid sank mehr herab, die Beweglichkeit war nach allen Seiten eingeengt und schmerzhaft. Gleichzeitig fühlte sich Patient sehr unwohl, schwindelig und müde; er hatte öfters Erbrechen und musste sich zu Bett legen. Es wurden kalte Umschläge gemacht. Darauf soll in einem Tag nicht nur der Schmerz aufgehört haben, sondern die ganze Vortreibung des Auges und die anderen Symptome geschwunden sein. Patient soll am anderen Tage angeblich wieder ganz wohl gewesen sein. Eine Verfärbung der Lider oder der Bindehäute habe er nicht bemerkt. Die jetzigen ganz akuten Erscheinungen sollten während einer Reaktion auf Kochsche Lymphe eingetreten sein. Am 4. II. hätten sich dieselben Erscheinungen wieder einzustellen begonnen und allmählich zugenommen. Als Patient in die Marburger Klinik aufgenommen wurde, hatte der Prozess bereits seinen Höhepunkt erreicht. Der Kranke war sehwächlich, sehr blass, seine Gesichtsfarbe fast grünlich, Lippen anämisch; Patient war benommen, einer Ohmmacht nahe. Dieselbe stellte sich auch nach kurzer Zeit ein. Nach dem Erwachen bestand Übelkeit, Schwindel, Erbrechen galligen Schleimes. Der Kranke fühlte sich sehr elend, Puls 56, Atmung nicht verlangsamt. Rechts mässiger Exophthalmus, Auge etwas nach aussen abgelenkt, in mässiger $\mathrm{Ab}$. duktionsstellung; Beweglichkeit nach allen Seiten eingeengt, am stärksten nach oben, unten und aussen, am wenigsten nach innen. Abducenslähmung bestand nicht; es erfolgten leichte Raddrehungen im Sinne der Obliqui. Ptosis incompleta, geringe Mydriasis, doch reagiert die Pupille, wenn auch etwas träger als links. Akkommodation normal. Ophthalmoskopisch Erwei terung und Schlängelung der Netzhautvenen, keine Pulsationen. Auch die dem Bulbus zunächst gelegenen Weichteile der Orbita schienen vorgetrieben; eine umschriebene Resistenz liess sich nicht palpieren. Passiv war der Bulbus nach allen Seiten sehr ausgiebig beweglich.

Bis nachmittags drei Uhr blieben die Symptome unverändert. 
Patient hatte öfters Erbrechen, heftige Kopfschmerzen. Dann stellte sich eine leicht bläuliche Sugillation der Haut des Unterlides im inneren Winkel ein. Dieselbe nahm an Ausdehnung zu, während der Exophthalmus und sämtliche andere Erscheinungen deutlich abnahmen.

Abends sechs Uhr war noch geringer, aber deutlicher Exophthalmus vorhanden, die Ptosis kaum mehr nachweisbar, die Beweglichkeit des Auges nach allen Seiten ausgiebiger, die Pupille noch etwas weiter, keine deutliche venöse Netzhauthyperämie mehr. Stellung des Auges ziemlich richtig. Innen war die Haut des Unterlides wallnussgross blaugrün sugilliert, die Conjunctiva nicht verändert. Bei Kompression der rechten Carotis trat keine sichtbare Änderung am Auge ein; auch subjektiv gab Patient rechts das gleiche Gefühl wie links bei Kompression an.

In den nächsten Tagen verringerte sich die Protrusion, welche beim Bücken regelmässig etwas zunahm. Die innere Hälfte des unteren Lides blieb noch Tage lang blau sugilliert. Am 13. XI. 91 und 15. XII. 91 wurde der Exophthalmus noch beim Bücken neben praller Schwellung der Lider bemerkt. S. war $=1$, die Akkommodation normal, die rechte Pupille noch etwas weiter, ihre Reaktion erhalten.

Über die Pathogenese des periodischen Exophthalmus à volonté bei meinem ersten Patienten vermag ich bei der nur oberflächlichen Untersuchung und Beobachtung desselben keine sicheren Angaben zu machen; besonders bin ich ausser stande die Insufficienz des R. superior auf dem rechten Auge richtig zu deuten. Man könnte vielleicht an einen kavernösen Tumor als Ursache der Beweglichkeitsbeschränkung dieses Muskels denken, indessen vermochte ich von einer Geschwulst hinter dem Auge nichts zu entdecken. Diese Annahme scheint mir ferner mit Rücksicht auf die sonst normale Stellung des rechten Auges nicht sehr wahrscheinlich zu sein - ich möchte vielmehr auch in diesem Fall eine variköse Venenerweiterung in der Orbita 
als Ursache des intermittierenden Exophthalmus beim Bücken beschuldigen.

Diese Ätiologie scheint mir aber in dem zweiten Fall über jeden Zweifel erhaben zu sein. Dass die Kur mit den Koch. schen Tuberkulininjektionen in einem direkten Zusammenhang mit dem intermittierenden Exophthalmus und den späteren Beobachtungen in der Marburger Augenklinik gestanden hat, möchte ich bezweifeln. Die retrobulbäre Hämorrhagie scheint mir ein Beweis dafür zu sein, dass damals bereits eine Dilatation und Verdünnung der Orbitalvenen bestand, welche hierdurch zum Bersten aus geringfügigem Anlass, vielleicht infolge eines Hustenanfalles, disponiert waren. Durch den Husten tritt ja bekanntlich eine Anschwellung der Venen des kleinen Kreislaufs ein; Donders hat ferner eine Ausdehnung der extra- und retrobulbären Venen bei forciertem kräftigem Exspirationsdruck unter normalen Verhältnissen nachgewiesen und gesehen, dass, wenn der Exspirationsdruck eine Zeit lang anhielt, der Augapfel nach vorn tritt. Wir können uns hiernach wohl vorstellen, dass bei bestehender Dilatation der Orbitalvenen und gleichzeitiger Verdünnung ihrer Wände während des Hustens die Ausdehmung so stark werden kann, dass ein Gefäss platzt und eine retrobulbäre Blutung erfolgt. Bei normaler Beschaffenheit der Venen dürfte eine solche Ruptur während des Hustens wohl kaum eintreten; nur beim Keuchhusten sind diese Gefüssrupturen beobachtet, hier sind die Hustenanfülle aber jedenfalls viel bedeutender, als sie es je bei unserem Kranken waron. Derselbe wusste sich nicht zu entsinnen in der betreffenden Nacht, als die Hämorrhagie entstanden war, einen stärkeren Hustenanfall gehabt zu haben. Er hat nur angegeben, dass er bereits seit einem Jahr zuvor an diffusen Schmerzen im rechten Auge gelitten und den Eindruck gehabt habe, dass ihm beim Bücken oder bei starken Anstrengungen das rechte Auge etwas vortrete.

Die retrobulbäre Hämorrhagie dürfte im Verein mit der 
Venenerweiterung die Veranlassung zu einem allmählichen Schwund des Orbitalfettzellgewebes und auf diese Weise später auch zur Ausbildung des Enophthalmus gewesen sein.

Für die Annahme einer kongenitalen Anlage zur Dilatation der Orbitalvenen scheint mir auch das Auftreten multipler kleiner teleangiektatischer Tumoren in der Haut über dem Sternum zu sprechen. Wenn ein kavernöser Tumor in der Orbita die Veranlassung für den intermittierenden Exophthalmus bei diesem Kranken wäre, so würde der unter gewöhnlichen Verhältnissen bestehende Enophthalmus geradezu unverständlich sein. Bei dem bereits mehrere Jahre bestehenden Zustand sollte man eher einen wenn auch nur geringgradigen Exophthalmus schon bei aufrechter Kopfhaltung erwarten, sobald ein Kavernom hinter dem Bulbus vorliegen würde.

Die starken Blutungen aus Nase und Mund sprechen ebenfalls für eine lokale Disposition der venösen Gefässwände zur Dilatation und zu spontanen Rupturen, speziell für eine abnorme Dünnheit derselben. Man dürfte deshalb nicht fehlgehen in der Amnahme, dass auch in den anderen Zuflüssen der V. ophthalmica superior und in ihr selbst eine abnorme Dünnheit und Nachgiebigkeit der Gefässwandung bestanden hat. Nach Gurwitsch gehören nämlich die Ethmoidalvenen zu den Zuflüssen der V. ophthalmica superior. Gerade bei der V. ophthalmica superior und ihren zahlreichen Aesten liegen die Chancen für eine Varikositätenbildung besonders günstig. Zunächst sind diese Gefässe nach den übereinstimmenden Angaben aller Autoren, welche sich mit ihrer Untersuchung beschäftigt haben (Seesemann, Merkel, Gurwitsch) klappenlos; ferner zeigt die $V$. ophthalmica superior in ihrem ganzen Verlauf vielfache natürliche Verbreiterungen und Verengerungen ihres Lumens, wie die Beschreibungen von Merkel und Gurwitsch ergeben, eine besondere Enge des Gefässes besteht z. B. vor ihrer Einmündung in den Sinus cavernosus, also an einer Stelle, an welcher auch 
Sergen $t$ besondere prädisponierende Momente für Strombehinderung des Blutes vermutet hat. Dass an diesem Ort thatsächlich Veränderungen eintreten können, welche ein Cirkulationshindernis und die Veranlassung zu Blutstauungen abgeben können, beweist die Litteratur. In einem Fall von Guthrie mit wahrem Aneurysma beider Artt. ophthalmicae war die V. ophthalmica cerebralis ebenfalls sehr erweitert und da, wo sie durch die Fissura orbitalis superior hindurchtritt, durch eine bedeutende Anschwellung von fast knorpelartiger Härte verschlossen, welche nach Mackenzie zum Vortreten des Auges ebenso viel beigetragen haben soll als die erweiterten Gefässe. An dieser Stelle der abnormen Verengerung der Vene kömnen leicht durch chronische Endophlebitis neue Stromhindernisse entstehen und $\mathrm{zu}$ einer Venendilatation führen.

Die variköse Erweiterung der Venen allein vermag indessen noch nicht das Zustandekommen des intermittierenden Exophthalmus zu ermöglichen; eine weitere Bedingung für das Hervortreten und Zurücksinken des Auges ist die Erschlaffung des Fascienapparates, durch welchen der Bulbus im vorderen Abschnitt der Orbita fixiert und in der Schwebe erhalten wird. Der Unterschied der Lage des Augapfels bei aufrechter und geneigter Kopfhaltung ist um so grösser, je tiefer unter gewöhnlichen Verhältnissen das Auge in der Orbita liegt. Fettschwund bedingt nicht nur einen gewissen Grad von Euophthalmus, den wir mehrfach in diesen Fällen angegeben finden; derselbe erleichtert im Zusammenhang mit der bei jeder Kopfneigung während der Arbeit eintretenden Protrusion des Auges die Auflockerung und Erschlaffung des Fascienapparates. Der Fettschwund ist eine Kompressionserscheinung, welche durch die abnorme Füllung der varikösen Venen während der Protrusion des Auges begünstigt wird.

$\mathrm{Zu}$ besonderen therapeutischen Massnahmen liat dieser $\mathrm{Zu}$ stand des intermittierenden Exophthalmus noch nicht geführt. 
Man hat Injektionen mit Ferrum sesquichloratum mit Erfolg gemacht, ja es ist schon darauf hingewiesen, dass die operative Beseitigung der varikösen Venen im gegebenen Fall bei strenger A- resp. Antisepsis nichts Bedenkliches haben dürfte; wird doch auch die doppelte Unterbindung und Exstirpation variköser Venen an den unteren Extremitäten mit Erfolg ausgeführt. Bei dieser Gelegenheit dürfte zuerst eine Bestätigung für die Annahme der varikösen Venenerweiterung als Ursache unseres Krankheitsbildes erhofft werden können, wenn uns nicht sonst zufällig die Sektion eines einschlägigen Falles die nötige Aufklärung bringt.

Nachfolgend gebe ich nun eine tabellarische Übersicht über 18 Fälle aus der Litteratur excl. meiner eigenen Beobachtungen. Bei 6 Patienten lag ein Tumor vor, bei den übrigen fehlte derselbe; $10 \mathrm{mal}$ wurde im letzteren Fall Enophthalmus konstatiert.

\section{Tabelle.}

\begin{tabular}{|c|c|c|c|c|c|c|}
\hline $\mathrm{Nr}$ & Autor & 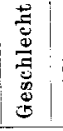 & 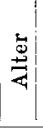 & $\stackrel{80}{3}$ & Entstehung & Symptome und Verlauf \\
\hline 1. & $\begin{array}{c}\text { Foucher et } \\
\text { Nélaton }\end{array}$ & W. & & L. & $\begin{array}{l}\text { Seit } 1 \text { Jahr schmerz- } \\
\text { loser Tumor im in- } \\
\text { neren Abschnitt des } \\
\text { oberen Augenlides } \\
\text { unterhalb des Augen- } \\
\text { höhlenrandes in der } \\
\text { Orhita }\end{array}$ & $\begin{array}{l}\text { Patientin litt von Jugend auf an } \\
\text { einer beträchtlichen Vergrösserung } \\
\text { der Schilddrüse. Bei aufrechter } \\
\text { Kopfhaltung war der Tumor nicht } \\
\text { sichtbar. Jidhaut normal, weder } \\
\text { Pulsation noch Tumor sichtbar. So- } \\
\text { bald die Patientin den Kopf bückte, } \\
\text { sah man sofort an der bezeichneten } \\
\text { Stelle eine Geschwulst von der } \\
\text { Grösse einer kleinen Wallnuss; die- } \\
\text { selbe war schmerzlos, weich, ein- } \\
\text { drückbar, fluktuierte, zeigte keine } \\
\text { Pulsationen, kein Gerüusch. Sie } \\
\text { vergrösserte sich bei starker Ex- } \\
\text { spiration und verkleinerte sich bei } \\
\text { tiefer Inspiration. Sie erschien bei } \\
\text { Kompression der Y. jugularis, spe- } \\
\text { ziell der linken Seite, wenn die } \\
\text { Frau weinte oder in Zorn geriet. } \\
\text { Der Tumor verschwand in einigen } \\
\text { Minuten beim Aufrichten des Kopfes. }\end{array}$ \\
\hline
\end{tabular}




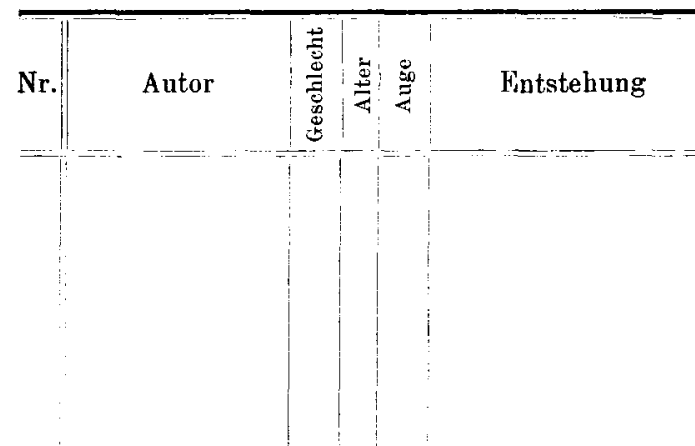

2. Mazel und A. M. 14 R. Vor 3 Jahren Wurf Boniface d'A nd uze

3. v. Grä fe mit Bovist

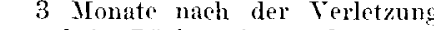
gegen den untern äus-! trat heim Bürken ein mandelgrosser sern Augenumfang 'Tumor im unteren Augenlid ohne

Das Auge war cin wenig in seinen Bewegungen behindert. Schnelle Frmüdung des Schens, wenn die Geschwulst ihren grössten lmfang erreicht hatte; Trübung desselben beim Versuch zn arbeiten. Völlige Heilung durch Injektionen von Liquor ferri sesquichlorati.

Schmerz und sehstörung anf, we]cher bei anfrechter Kopfhaltung wieder verschwand. Der Tumor war wejch, hatte blaue Farbe, pulsierte nicht und liess sich sogleich zurückdrücken. Ange und Sehvermögen waren normal. Bei rechter Seitenlage wurde die Ceschwulst grösser, bei linker Seitenlage kleiner, verschwand aber nicht ganz. Bei aufrechter Stelhung füllte man an Stelle der für gewöhnlich nicht sichtbaren Geschwulst cine Vertiefung in dem normal gefärbten unteren Lide, in welche man bequem die Zeigefingerspitze eindrïcken konnte; die Delle verschwand nur langsam und nuvollständig. Erst beim Bürcken wurde der 'Tumor sichtbar, gleichzeitig drängte er den Bubus etwas nach cinwïrts; die Lidhatut ersehien dabei bläulich verfärlot.

W. 30. R. Die gravide Patien- L'nter dem wberen lid fand sich tin bemerkte jüngst eine halhhusehussgressie, kirschrote beim Bücken, dass Geschwulst temporalwaits hinter der cine Geschwulst am gesunden Übergangsfalte. Nie war ausseren Augenwinkel weich, kompressibel und verschwand zwischen den Lidem bei leisestem Druek. An ilurer Oberherrortrat und beim fläche zeigten sich hart unter der Anfrichtenwiederver- Bindohaut mehrere knollenfömig schwind

zusammengew undene Gefïsse. Sie pulsierte nicht, war aber ausserordentlich sehwellbar. Das Hervortreten des Tumors beim B̈̈̈cken rührte teils von einer Vemelnung seines ejgenen Volumens, teils von der venören seliwellung des Orbitalfett zellgerebes hor; es erfolute auch bei Kompression der Jugular- 


Nr. Autor

venen. v. Griffe hielt die Geschwulst für cine angeborene, aher unter dem Einfluss der Gravidität vergrösserte Teleangiektasie.

4. E. Grüining

Mr. 45 R. Vor 5 Jahren beim In aufrechter Stellung erschien Legen eines Teppichs Patient etwas glotzäugig, rechts in knieender und vorn- mehr als links; bei Rückenlage übergeneigter Stellung erhob sich das rechte Auge nicht Gefühl von Schwere über das Niveau des linken. Beide und Hervortreten des: Augen nomal beweglich. R. Mp.

$\begin{aligned} & \text { Auges, das bei Be- } \\ & \text { sichtigung im Spiegel }\end{aligned} \frac{1}{4^{1 / 2}} \mathrm{~S}=20 / 50$, L. Mp. $1 / 6 \mathrm{~S}=$ später nicht wahrge $20 / 70$; Jäger 1 . Papillen weiss und nommen werden atrophisch; beiderseits ringförmiges konnte. Seit der Zeit Staphyloma posticum, Arterien dünn, regelmässig beim Venen geschlängelt. Gesichtsfeld Bücken Vorfall des frei; grün für rosa gehalten, sonst Auges: Farben normal. Keine Struma, Herz normal. Beim Neigen des Kopfes allmählich zumehmende Vortreibung des r. Auges, das fast unbeweglich wird, geringe Abduktionsfähigkeit, aber schmerzhaft. Bulbus lïsst sich leicht zurückdrücken. Carotiskompression ist ohne Einfluss auf den Exophthalmus. Kein Gerïusch hörbar. Kn a pp nahn an, dass der Exophthalmus von einem ahnorm erweiterten, beim Bücken sich füllenden Venenkonvolut des Orbitalzellgewebes abhängig wäre.

5. Viensse

M. L. Vor etwa 4 Jahren

Soldat des 34 . Artillerieregiments. während ciner meh- Bei aufrechter Haltung Enophthalrere Minuten danern. mus, beim Bücken Exophthalmus den Ohnmacht $\mathrm{Ex}-\mathrm{and}$ Blindheit nach einigen Sekunophthalmus bemerkt den. Lider angeschwollen, gedunsen. Bindehaut kongestioniert. Keine Pulsation. Auge sonst normal. Beim Bücken kein Schmerz, keine Ernuïdung. Keine Hirndrucksymptone bei Druck auf den in die Orbita zurücksinkenden Bulbus. Vieusse glaubte, dass es sich um eine ausgedehnte angeborene Kommunikation des Arachnoidalraumes mit der Tenonsehen Kapsel durch die Fissura orbitalis superior handele. Der Referent des $\mathrm{N}$ a ge lschen Jahresberichts weist aber diese Erklärung zurück und hält eine venöse Gefässgeschwulst in der Tiefe der Orbita für wahrscheinlieh. 


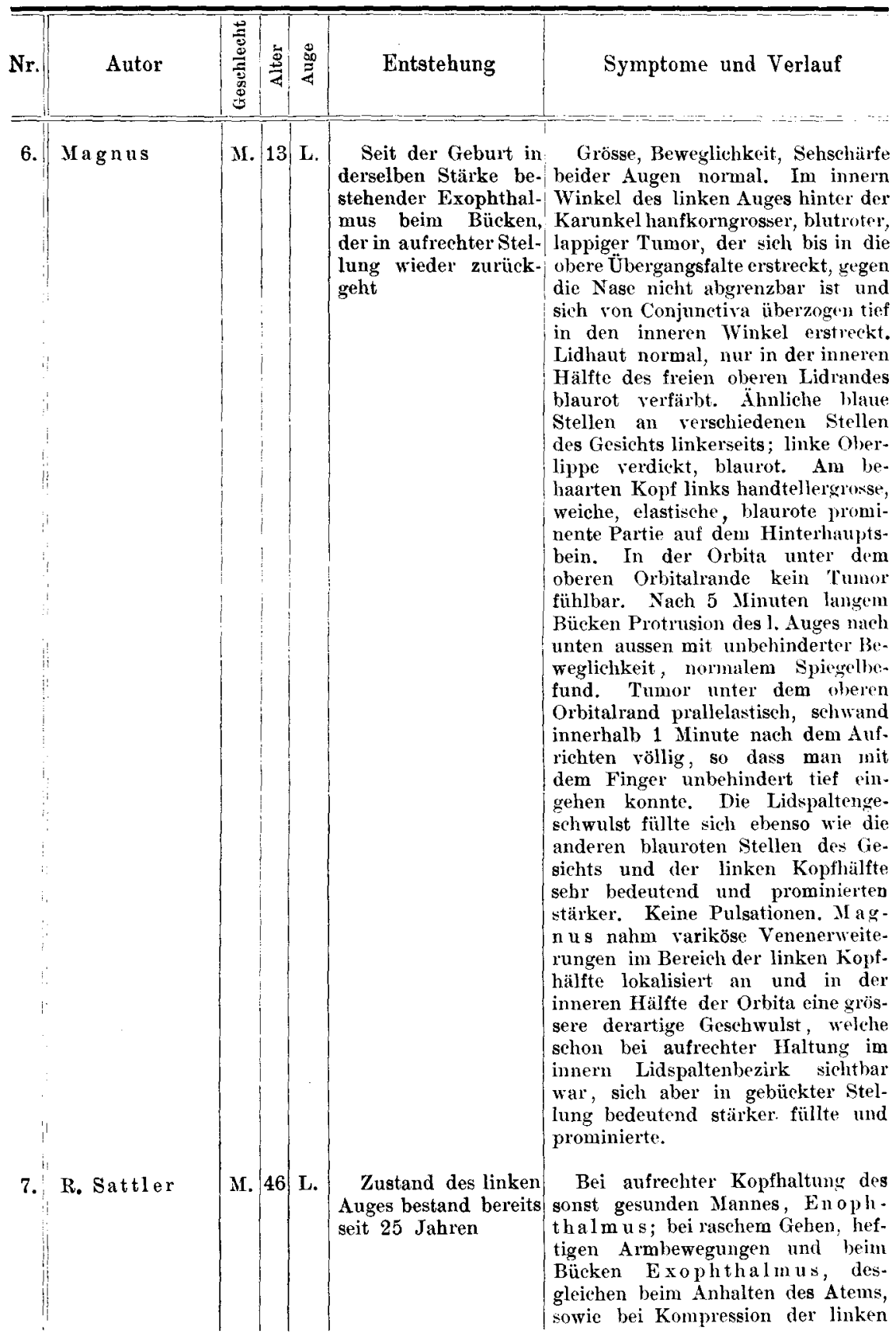




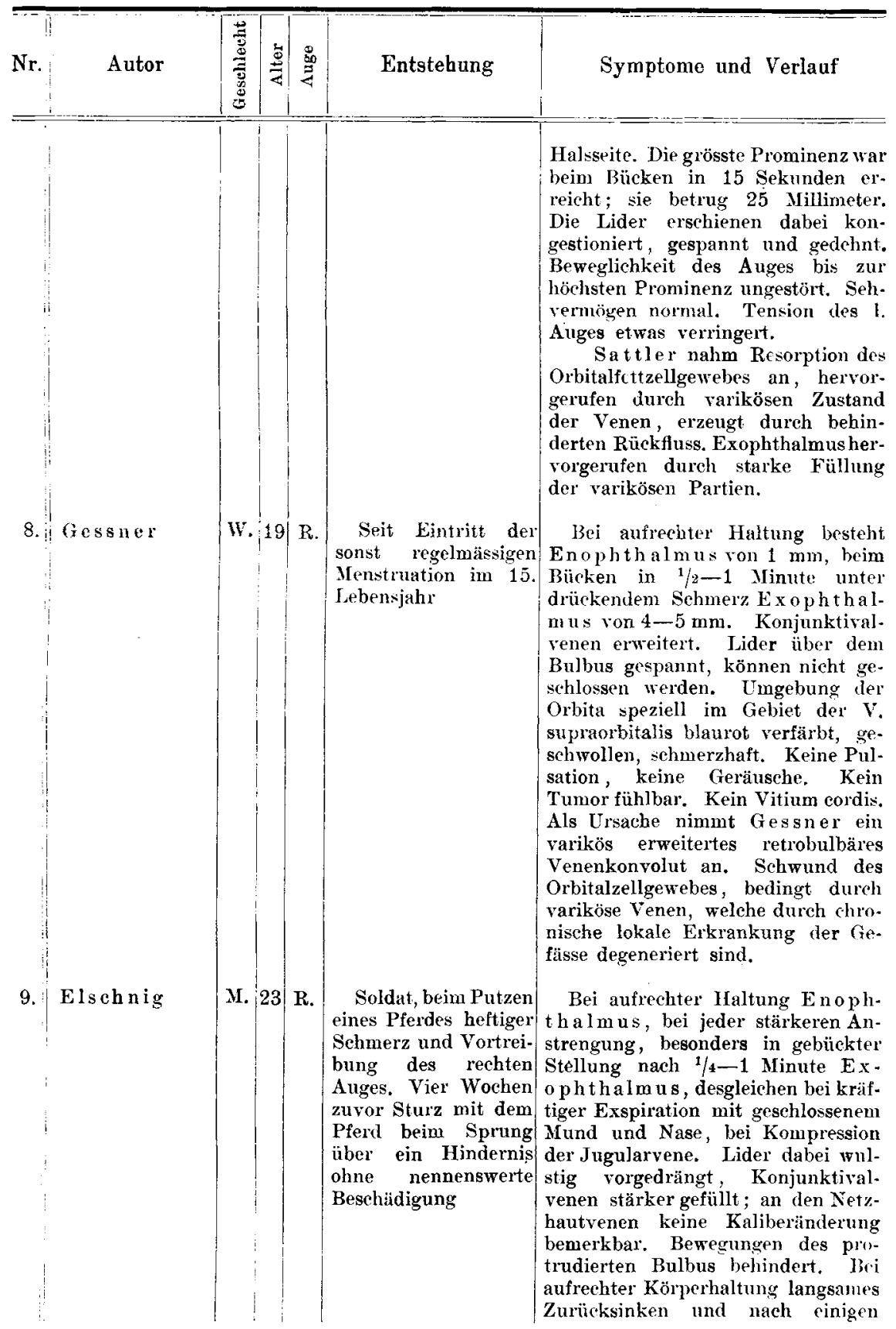




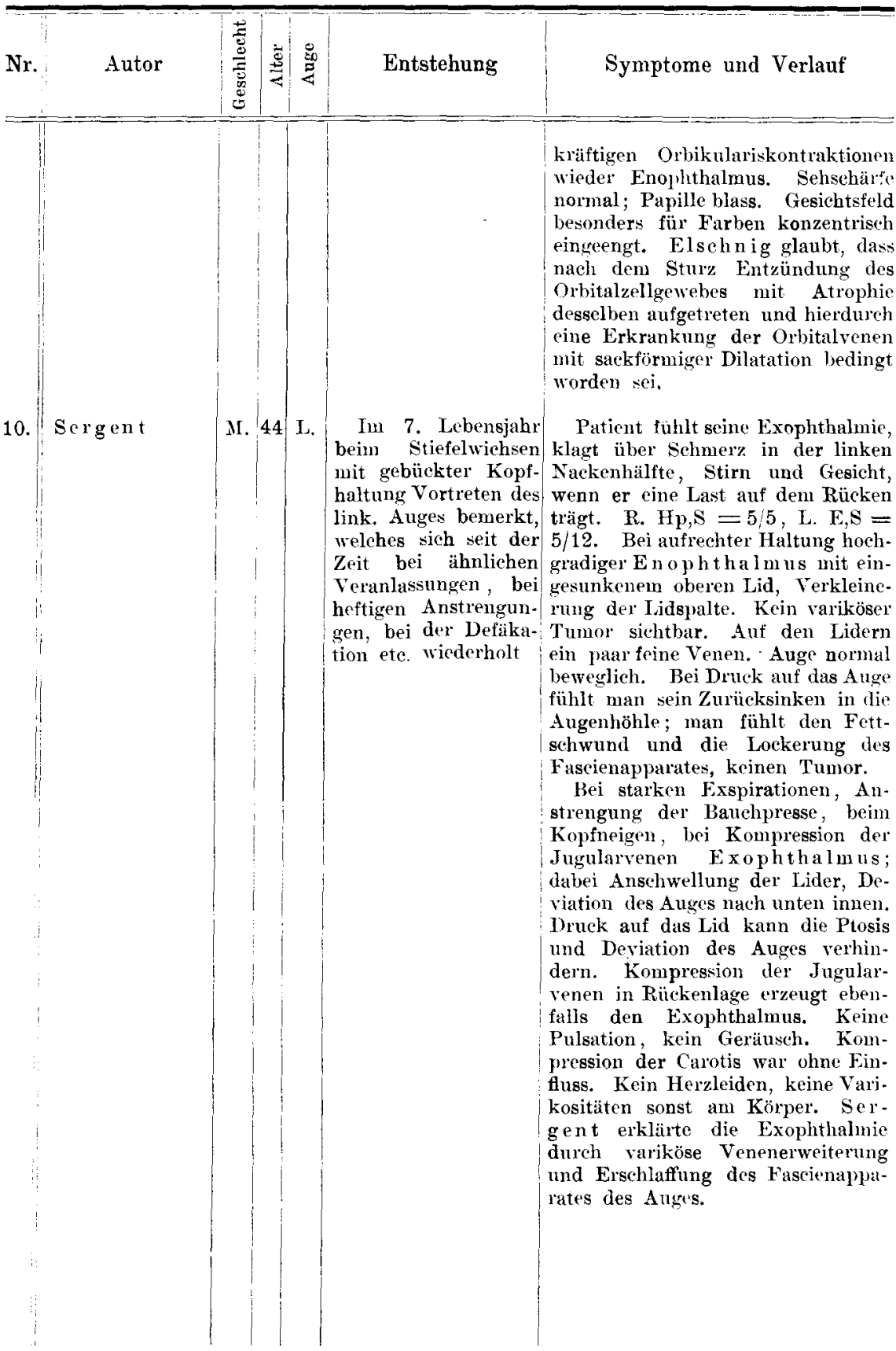




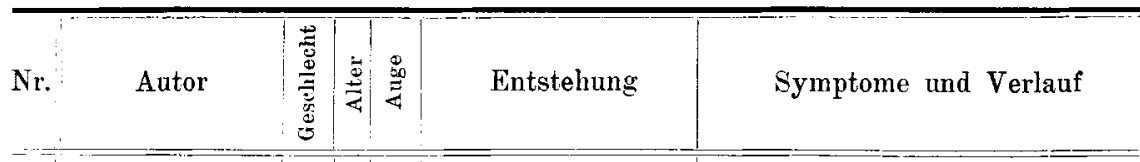

\begin{tabular}{|c|c|c|c|c|}
\hline 11. Panas & W. 38 & L. & $\begin{array}{l}\text { In Altry ron } 18 \\
\text { Jahren Stoss an der } \\
\text { linken Augenbrauen- } \\
\text { gegend. Leichter Ex- } \\
\text { ophthalmus. Seit } 10 \\
\text { Jahren Tumor im } \\
\text { oberen inneren Augen- } \\
\text { winkel, sobald sich } \\
\text { die Kranke bückt oder } \\
\text { artrengt. } 5 \text { Jahre }\end{array}$ & $\begin{array}{l}\text { Enophthalmus, beim Bücken } \\
\text { starker Exophthalmus, venöser } \\
\text { Tumor in der inneren Hälfte des } \\
\text { oberen Lides. Kein Schmerz. Seh- } \\
\text { vermögen normal. Strabismus con- } \\
\text { vergens concomitans dexter. }\end{array}$ \\
\hline
\end{tabular}
später im Verlauf einer Grippe Ekchymosen im Lide. 1 Jahr spitter Enophthalmus
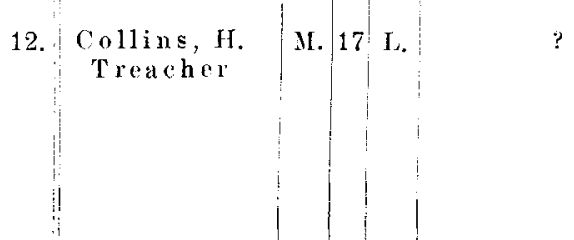

13. Riehter, P. V. W. 14 R. Seit ca. 1 Jahr

Leichter Enopht halmus, heim Bücken nach 1 Minute Exoph. thal nus, die Netzhautvenen sehr erweitert und pulsierend. Collins nimmt eine Gefüsshöhle in der $\mathrm{Or}^{\mathrm{r}}$ hita an, welche sich beim Bücken fïllt.

Vortreten des recht. links. Lid- und BulbusbeweglichAuges ohne irgend keit normal, $S=1$. Spiegelbefund welche Belästigung beiderseits normal.

beim Bücken. Kein Exophthalmus rechts beim Neigen Trauma, keine stär- des Oberkörpers nach 1 Hinute bei kere körperliche An- mässiger Rötung der Wange und strengung. Im Laufe Stirn; deutlich sichthare temporale des nächsten Jahres| Venen. Oberlid etwas ödewatös, hat die Protrusion! bedeckt die Cornea, Bulbus pronoch etwas zugenom- trudiert und nach aussen unten dismen, dann blich sie loeiert. Nach 1/4 Minute kehrt im stationär

Aufrichten das Auge in seine nol. male Lage zurück, schwillt das Oberlid ab. Während der Exophthalmie Lidschluss erschwert, Conjunctiva injiziert, Geschwulst nieht fühllbar, keine Pulsation, kein Gcräusch. Exophthalnie tritt auch rin bei Kompression der rechten $v$. jugularis, in geringem Grade auch bei Druck auf die linke V. jugularis, am stärksten bei gleichzeitiger Kompression beider Venen. Fernex Exophthalmus bei forcierter Fxspiration, während Mund und Nase gesehlossen werden, desgleichen bei heftigem Hin- und Herschleudern des Kopfes. Kompression der Carotis ohne Einfluss. Einen Tag 


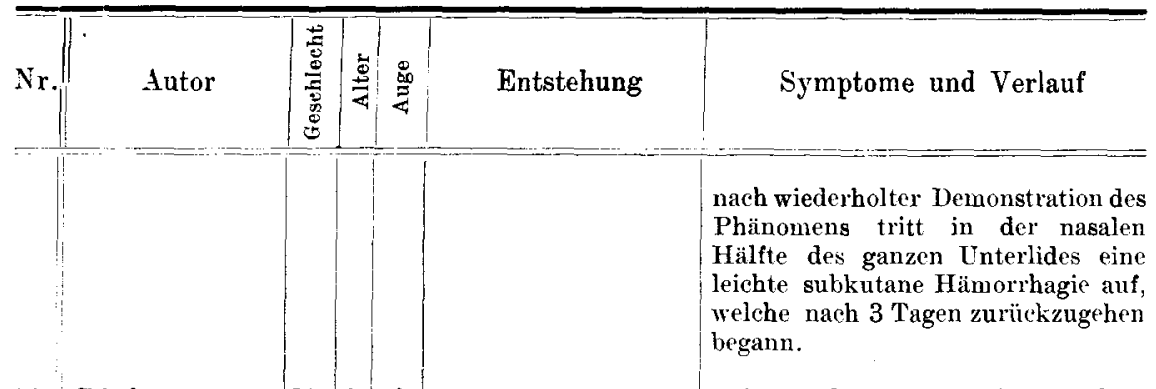

14. Riehter

M. ? ?

Seggel hatte bei einem Soldaten, welcher an Varikosititen der unteren Extremitäten litt, Exophthalmus beobachtet, welcher zum erstenmal bei einer starken Anstrengung während des Fechtens aufgetreten war.

15. van Duyse u. M. 29 R. Bribosia
Vor 1 Jahr Stossge- Häufiger En ophthalu us von gen die rechte Schläfe ca. $4 \mathrm{~mm}$, keine varikösen Venen mit Blutunterlaufung in den Lidern oder ihrer Lingebung. der Lider, 2 Monate Keine Bewegungsstörung des Auges, lang Nachts Schmer- beim Blick aufwärts Nystagmus. zen. Seit der Zeit Bei jeder Anstrengung, beim Bücken regelmässig beim Bük- tritt innerhalb 1 Minute Exophken oder bei stärkeren thalmus mit Ablenkung des Augres Anstrengungen Pro- nach aussen und unten ein, gerintrusion des r. Auges; ges Herabsinken des oberen Lides, dabei siehter nicht und mässige Erweiterung der Pupille. hat heftige Schmerzen Bei Kompression der Jugularvenen, um dic Augenhöhle bei jeder intravaskulären Drucksteigerung in den Kopfvenen Vortreibung des Auges, Diplopie ; Schwächung des Sehvermögens wïhrend der Protrusion, Hintergrundgefässe normal. Man fühlt keinen Tumor, keine P'ulsation, hört kein Geräusch. Am Körper keine Varicen. Der höchste Grad von Exophthalmus tritt ein, wenn Pat. bei angehaltenem Atem sich anstrengt, im Mittel nach 30 Sekunden; es dauert einige Sekunden mebr, bis das Auge seine frïhere Stellung wieder erlangt. In Rückenlage tritt der Bulbus nicht tiefer in die Augenhöhle; $\mathrm{er}$ springt rielmelu etwas vor nach aussen und unten - Diplopie mit Höhenunterschied der Doppelbilder. In rechter Seitenlage springt das Ange noch mehr ror mit entsprechender Diplopie, die auch in Bauchlage nachweisbar ist.

Die Autoren nehmen eine variköse Venenerweiterung als Ursache des Ieidens an, welche hervorge- 


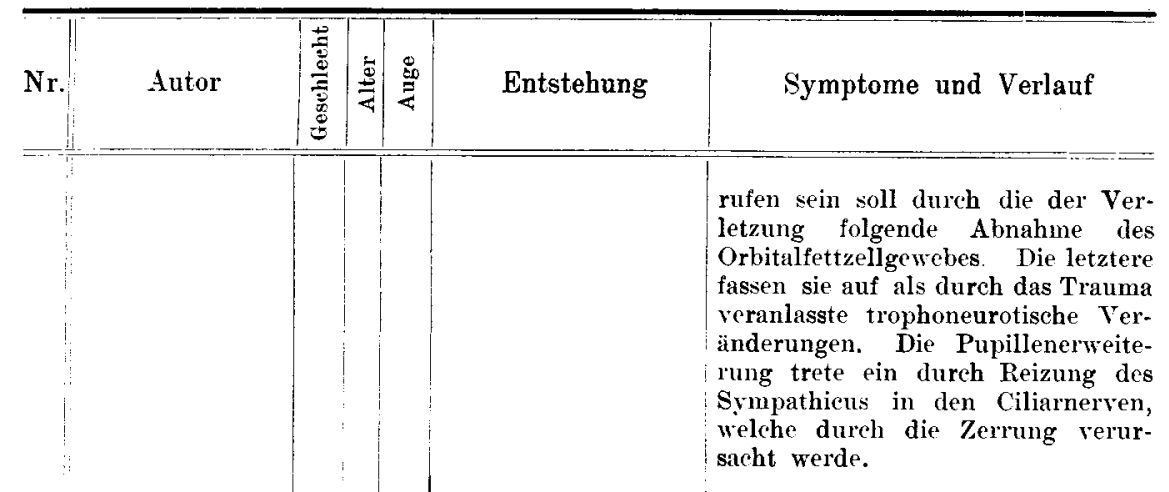

16. Mayer, L.

17. Teroon, Jean, M. 21 R.
Vor 12 Jahren beim Bücken Hervortreten rechten des (im venösen Auges. Keine Ver- Stromgebiet. Herz und innere Orletzung, hat frïher gane normal. Links geringe Ptosis; viel Feldarbeit in ge- Enophthalmus. Beweglichkeit bückter Stellung aus- der Lider und des Auges normal. führen müssen

R. Hp. 1 DS $=1$; Presbyopie. L. Optikusatrophie ohne Zeichen von Entzündung; Venen stärker gefüllt. Regelmässig beim Bücken nach 1 Minute Exophthalmus; bei längerem Bücken tritt Flimmem vor den Augen und Schwindelgefiihl ein. Gesicht dabei mïssig gerötet; oberes Lid ödematös geschwellt, bedeckt den Bulbus ganz. Temporale Venen stark gefüllt. Bulbus nach oben und aussen gedreht. Äusserlich keine Geschwulst, keine Pulsation fühlbar; kein Geräusch. Während des Exophthalmus leichte ziehende Schmerzen im linken Auge. Nach dem Aufrichten sofort wieder Enophthalmus. Exophthalmus wird auch hervorgerufen durch Kompression der linken $V$. jugularis oder beider Venen. Kompression der Carotis ohne Einfluss, Der Autor nimmt variköse Entartung der retrobulbären Venen wie sekundäre Druckatrophie des Orbitalfett. zellgewebes an und erwägt die operative Beseitigung nach doppelter Unterbindung.

Seit 3 Monaten be- Bei der Defükation, beim Coitus, stand das Leiden, ohne bei jeder Anstrengung und bei der dass eine Verletzung Kompression der Jugularvenen trat vorangegangen war. Exophthalmus auf; dabei erweiterWithrend Patient beim ten sich die Venen etwas. Der schreiben den Kopf Augenhintergrund und die Sehschärfe 


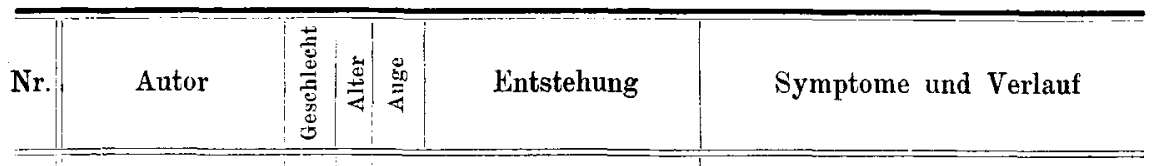

beugte, bemerkte er des rechten, leicht myopischen Auges plötzlich ein Herror- (2 Dioptr.) waren normal. Fïr ge. treten des Anges. wöhnlich bestand ein geringer Grad ron Enophthalmus ohne Ptesis. Dic Pupille des rechten Auges war etwas weiter als die des linken; beide reagierten gut. Während der Enophthalmie fühlte man nichts Besonderes in der Orbita, während der Exophthalmie einen Widerstand, aber keinen Tumor. Weder an den Lidern noch im Gesicht fanden wich dilatierte Venen.

Der kleine, hlasse und schwitchliche Kranke war Neurastheniker; er hatte seit 3 Jahren Hämorrhoiden; keine Varicen an den Beinen. Herz war normal.

In Übereinstimmung mit van Du y se glaubt 'Terson, dass die Enophthalmie die Folge eines Gewebsschwundes in der Orbita durch Trophoneurose: sei und auf einer Sympathicusaffektion basiere. Fr bezeichnet nach dem Vorgange seines Bruders A. Ters o n das Leiden als „Enophthalmie et . Exophthalmie alternantes", weil dadurch die Aufeinanderfolge der beiden Hauptsymptome am besten ausgedriickt verde; der Name „Exophthalmie

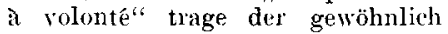
vorhandenen Enophthalmie keine Rechnung. Den Fxophthalmus erklärt er dureh Dilatation und stiarkere Füllung der Venen der Orbiti.

In seiner übrigens nicht vollständigen Litteraturübersicht führt Terson noch einen Fall (18) von Schwarzschild an, welchen ich im Original nicht erhalten konnte. Bei einem 20jährigen Kutscher hatte sich im Anschluss an einen Schlag auf den rechten Augenhöhlenrand mit Wunde in der Augenbrauengegend ein Enophthalmus von $6 \mathrm{~mm}$ und ein Einsinken des obern Augenlides mit Verkleinerung der Lidspalte ausgebildet. 
Wenn er bei aufrechter Körperhaltung den Kopf beugte, trat Exophthalmie von $6 \mathrm{~mm}$ ein. Beweglichkeit des Auges war nahezu erhalten, Sehvermögen und Augenhintergrund normal. Die rechte Gesichtshälfte war in ihrer Entwickelung zurückgeblieben. Panas, welcher diesen Fall in seinem Lehrbuch kurz referiert, ist der Ansicht „qu'il s'agissait ... d'exophthalmie variqueuse".

Schliesslich sei noch ein Referat über eine kürzlich stattgefundene Demonstration eines einschlägigen Falles mitgeteilt. Nach Pergens zeigten Moyart und van Duyse in der zweiten Sitzung der Société Belge d'ophthalmologie zu Brüssel am 24. April d. Js. eine Frau, bei welcher „Varices der Venae ophthalmicae" des linken Auges vorhander waren. Das Auge war etwas hervorgetrieben. Bog die Frau den Kopf nur eine halbe Minute nach unten, dann wurde die Hervortreibung stärker, es entstand Ptosis und beschränkte Bewegung der äusseren Augenmuskeln; das Auge sah dann nicht mehr. Der ophthalmoskopische Befund blieb negativ. Dieser Zustand kehrte bald wieder zum vorherigen Status zurück; objektive oder subjektive Geräusche waren nicht zu bemerken.

Überblicke ich noch einmal kurz die in vorstehender $\mathrm{Ab}$ handlung aufgeführten klinischen Beobachtungen, so möchte ich meinen Standpunkt in der Frage des intermittierenden Exophthalmus dahin präcisieren, dass dieses eigenartige Krankbeitsbild, bei dem sich in der Mehrzahl der Fälle ein Tumor hinter oder neben dem Bulbus nicht nachweisen lässt, nach Massgabe des Vortreibens beim Bücken resp. ähnlichen Bedingungen und des Zurücksinkens des Auges beim Aufrichten des Körpers resp. bei Rückenlage sich füglich nicht anders als durch Erschlaffung des Fascienapparates des Bulbus und durch ein Moment erklären lässt, bei dem hinter dem Bulbus ein An- und Abschwellen des anatomischen Stützgewebes in der Orbita unter jenen Bedingungen möglich ist. Eine derartige An- und Abschwellbarkeit 
kann nur bei den Orbitalvenen erfolgen; dafür sprechen die physiologischen Beobachtungen von Donders, von Cohn. Die variable Füllung der Orbitalvenen muss natürlich viel hochgradiger ausfallen, wenn die Venen varikös entartet sind; dass dies beim intermittierenden Exophthalmus der Fall ist, lässt sich aus dem klinisch erwiesenen Vorkommen venöser variköser Tumoren folgern. Auch durch anatomische Untersuchungen ist das Vorkommen variköser Venenektasien in der Orbita festgestellt. Ihr Vorhandensein ist mehr als wahrscheinlich, wenn im gegebenen Fall sich Varicen oder Teleangiektasien auch noch an anderen Körperstellen, an den Extremitäten, am Rumpf, oder in der Umgebung des Auges vorfinden. Für die Venenektasien in der Orbita muss man eine lokale kongenitale Prädisposition an. nehmen. Zur Begünstigung der Venendilatation tragen die anatomischen Eigentümlichkeiten der V. ophthalmica superior voraussichtlich in hohem Grade bei, ferner zum Vortreten des Auges aus der Orbita dauernde Anstrengung und Arbeit in gebückter Stellung, Hämorrhagien in die Orbita, Verletzungen der Umgebung der Orbita, bei denen möglichenfalls Veränderungen in der Fissura orbitalis superior geschaffen werden, welche die Stauung des Blutes in den Orbitalvenen begünstigen. Ist der Exophthalmus erst einmal eingetreten, so wiederholt er sich später bei passender Gelegenheit immer wieder; es entsteht ein circulus vitiosus in An- und Abschwellen der Orbitalvenen, welcher zu Abnahme des Fettpolsters der Orbita und zu Enophthalmus bei aufrechter Körperhaltung führt. Ob bei dem Schwund des Fettpolsters trophische Störungen infolge einer Affektion des Sympathicus im Spiele sind, wie van Duyse und Terson annehmen, lasse ich dahingestellt. Die geringgradige Ptosis kann durch das Zurücksinken des Augapfels zu stande kommen. Ob die Erweiterung der Pupille durch Zerrung der in den Ciliarnerven verlaufenden Sympathicusfasern veranlasst wird, vermag ich nicht zu entscheiden; die Möglichkeit ist nicht abzuleugnen. 


\section{Litteratur.}

1. Merkel, Fr., Handbuch der topographischen Anatomie. Bd. 1. Braunschweig $1885-1890$.

2. Weiss, L., Beiträge zur Anatomie der Orbita. Heft 1. Tübingen 1888.

3. Cohn, H., Messungen der Prominenz der Augen mittelst eines neuen Instrumentes, des Exophthalmometers. Zehenders klin. Monatsblätt. f. Augenheilkunde, Bd. V, pag. 339-351.

4. Himly, E. A. W., Die Krankheiten und Missbildungen des menschlichen Auges und deren Heilung. Bd. I, pag. 393 und 394. Berlin 1843.

5. Arlt, F., Die Krankheiten des Auges. Bd. 3, pag. 178. Prag 1856.

6. Nieden, A., Zwei seltenere Fälle von Orbitalerkrankungen. Zehenders klin. Monatsbl. f. Augenheilkunde Bd. XIX, pag. 72-75.

7. Donders, F. C., Über die Stützung der Augen bei Blutandrang durch Atmungsdruck. Gräfes Arch. f. Ophthalmol., Bd. XVII, H. 1, pag. 80 bis 106.

8. Sattler, H., Pulsierender Exophthalmus. Gräfe-Sämisch, Handbuch der Augenheilkunde, Bd. VI, pag. 879. (Variköse Venengeschwülste in der Orbita.)

9. Andreae, A., Grundriss der gesamten Augenheilkunde. Teil II, pag. 145. Magdeburg 1837.

10. Fischer, J. N., Lehrbuch der gesamten Entzündungen und organischen Krankheiten des menschlichen Auges etc. Prag 1846, pag. 361.

11. Schön, M. J. A., Handbuch der pathologischen Anatomie des menschl.

- Auges. Hamburg 1828.

12. Stellwag v. Carion, Die Ophthalmologie vom naturwissenschaftlichen Standpunkt. Bd. II, H. 2, pag. 1275. Erlangen 1858.

13. Dup ont, Des tumeurs de l'orbite formées par du sang en communication avec la circulation veineuse intracranienne. Thèse de Paris 1865 .

14. Dictionnaire de Médecine ou Repertoire général des sciences medicales. Deuxieme Edition. XXX Tomes. Paris 1840. Article Maladies de l'orbite Velpeau, Tome XXII, pag. \$10. 
15. Foucher, Sur une variété de tumeur de l'orbite. Gazette des hôpitaux. 1858, Nr. 141.

16. Mazel et A. Boniface d'Anduze, Tumeur veineuse réductible de la paupière inférieure. Union medic. 1861, pag. 163. refer. d'Annales d'Oculistique, Tome XLV, pag. 273.

17. v. Gräfe, Vereinzeltes über Tumoren. Gräfes Arch. f. Ophthalmol. Bd. XII, H. 2, pag. 222.

18. Grüning, Exophthalmus dexter bei Vorwärtsbewegung des Körpers. Arch. f. Augen- und Ohrenheilkunde v. Knapp-Moos, Bd. III, pag 168.

19. Vieusse, Communication entre la cavité arachnoïdienne et la capsule de Tenon. Recueil d'Ophthalmol. 1878, pag. 314, ref. in Hirschbergs Centralbl. f. Augenheilkunde 1878, pag. 312 und in $\mathrm{Nagel}$ s Jahresbericht 1879, pag. 437 .

20. Magnus, H., Periodischer Exophthalmus sinister bei Beugen des Kopfes. Zehend. Klin. Monatsbl. f. Augenheilkunde 1889, pag. 62.

21. Sattler, R., A case of one-sided transitory exophthalmus with indisturbed function and muscular movements of the eye and the coexistance of enophthalmos or recession of the globe. Americ. journ. of med. sciences N. S. LXXXIX, 1885, pag. 486, ref Michels Jahresbericht 1885, pag. 485.

22. Gessner, C., Enophthalmos dexter übergehend in Exophthalmus bei Vorwärtsbeugen des Kopfes. Hirsch bergs Centralb]. f. Augenheilkunde 1889, pag. 161 .

23. Elschnig, A., Allgemeine Wiener med. Zeitung, Bd. XXXVII: 1892, pag. 61, ref. Hirschbergs Centralbl. f. Augenheilkunde, 1892, pag. 558.

24. Sergent, E., De l'Exophthalmos intermittent ou exophthalnie à volonté. Gazette des Hopitaux, 1893, Nr. 60.

25. Le Moine, Observation de tumeur variqueuse; naevus de le paupière supérieure; cauterisation ignée. Recueil d'Ophthalmol., 1887, pag. 416; ref. Revue générale d'ophthalmòl., 1887, pag. 475 und Michels Jahresbericht 1887 , pag. 433 .

26. Chisolm, J. J., Eine variköse Geschwulst des untern Lides, unsichtbar bei aufrecht gehaltenem, sehr gross bei gesenktem Kopf. Arch. f. Augenheilkunde von Knapp-Schweigger, 1891, Bd. 22, pag. 261-264.

27. van Santon, Spontane Hämorrhagie in de Orbita. Nederl. Tijdschr. v. Geneesk. Afd. 1, Nr. 3, ref. Nagels Jahresbericht 1872, pag. 427.

28. Gruss, Wiener med. Presse, 1887, Nr. 25, pag. 884 .

29. Yvert, Des tumeurs de l'orbite en communication directe avec la circulation intracranielle. Recueil d'Ophthalmol. 1881, pag. 1 und 93; ref. Michels Jahresbericht 1881, pag. 453.

30. De Vincentiis, C., Sull esoftalmo da neoplasia dell' orbitada ematoma orbitario-pulsante spontaneo e traumatico etc. Lavori della clinica ocul. della R. univ. di Napoli, Vol. IV, Fase. I, 1894. ref. Michels Jahresbericht f. Ophthalmol. 1894, pag. 522 .

31. Dolgenkow, W., Tumor cavernosus orbitae sin. Westnik ophth. 1886. ref. Michels Jahresbericht 1886, pag. 473. 
32. Jocqs, R., Contribution au diagnostic des tumeurs vasculaires de l'orbite. Clinique ophthalmologique, Mai 1895.

33. Idem, Sur un nouveau cas de dilatation veineuse de l'orbite. Clinique ophthalmulogique Aout 1895.

34. Collins, H. Treacher, A case of enophthalmos becoming exophthalmos on stooping. Transact Ophthalmological Society, Vol. XV, 1895. ref. Revue générale 1896 , pag. 379 .

35. Richter, P. V., Über intermittierenden Exophthalmus. Arch. f. Augenheilkunde von Kn app-Schweigger, Bd. 31, pag. 31-44, 1895.

36. van Duyse, Daniel et Bribosia, Edmond, Enophthalmos avec exophthalmie intermittente à volonté. Arch. d'Ophthalmol. v. P a n a s etc., Bd. XV, pag. 159-170, 1895.

37. Mayer, Ludwig, Ein Fall von Exophthalmus intermittens. Inaug.Diss. München 1896.

38. Moyart und van Duyse, Zehenders klin. Monatsbl., 1897, pag 168.

39. Terson, Jean, De l'Enophthalmie et de l'Exophthalmie alternantes. Paris 1897.

40. Schwarzschild, H., A case of palimtosis alternating with proptosis following injury. Med. Record XII, pag. 544 ref. in Panas traité des maladies des Yeux, T. II. Art. Enophthalmie. 